\title{
Quasar Selection Based on Photometric Variability
}

\author{
C. L. MacLeod ${ }^{1}$, K. Brooks ${ }^{1}$, Ž. Ivezić ${ }^{1}$, C. S. Kochanek ${ }^{2,3}$, R. Gibson ${ }^{1}$, A. Meisner ${ }^{1}$, \\ S. Kozłowski ${ }^{2,4}$, B. Sesar ${ }^{1}$, A. C. Becker ${ }^{1}$, W. H. de Vries ${ }^{5}$
}

Received __; accepted _ _

\footnotetext{
${ }^{1}$ Department of Astronomy, University of Washington, Box 351580, Seattle, WA 98195 (cmacleod@astro.washington.edu)

${ }^{2}$ Department of Astronomy, The Ohio State University, 140 West 18th Avenue, Columbus, $\mathrm{OH} 43210$

${ }^{3}$ The Center for Cosmology and Astroparticle Physics, The Ohio State University, 191 West Woodruff Avenue, Columbus, OH 43210

${ }^{4}$ Warsaw University Observatory, Al. Ujazdowskie 4, 00-478 Warsaw, Poland

${ }^{5}$ University of California, One Shields Ave, Davis, CA 95616
} 


\begin{abstract}
We develop a method for separating quasars from other variable point sources using SDSS Stripe 82 light curve data for $\sim 10,000$ variable objects. To statistically describe quasar variability, we use a damped random walk model parametrized by a damping time scale, $\tau$, and an asymptotic amplitude (structure function), $\mathrm{SF}_{\infty}$. With the aid of an SDSS spectroscopically confirmed quasar sample, we demonstrate that variability selection in typical extragalactic fields with low stellar density can deliver complete samples with reasonable purity (or efficiency, E). Compared to a selection method based solely on the slope of the structure function, the inclusion of the $\tau$ information boosts $E$ from $60 \%$ to $75 \%$ while maintaining a highly complete sample (98\%) even in the absence of color information. For a completeness of $C=90 \%, E$ is boosted from $80 \%$ to $85 \%$. Conversely, $C$ improves from $90 \%$ to $97 \%$ while maintaining $E=80 \%$ when imposing a lower limit on $\tau$. With the aid of color selection, the purity can be further boosted to $96 \%$, with $C=93 \%$. Hence, selection methods based on variability will play an important role in the selection of quasars with data provided by upcoming large sky surveys, such as Pan-STARRS and the Large Synoptic Survey Telescope (LSST). For a typical (simulated) LSST cadence over 10 years and a photometric accuracy of $0.03 \mathrm{mag}$ (achieved at $i \approx 22$ ), $C$ is expected to be $88 \%$ for a simple sample selection criterion of $\tau>100$ days. In summary, given an adequate survey cadence, photometric variability provides an even better method than color selection for separating quasars from stars.
\end{abstract}




\section{Introduction}

The selection of large samples of quasars, or active galactic nuclei (AGN), is a valuable tool in many areas of study, including galaxy evolution and black hole growth (Kollmeier et al. 2006), absorbing systems in the intergalactic medium (Hennawi \& Prochaska 2007; McDonald \& Eisenstein 2007), and determining the large scale structure of the universe (Ross et al. 2009). Extending these studies to significantly fainter quasars at any given redshift is a major goal for the next-generation Large Synoptic Survey Telescope (LSST; Ivezić et al. 2008). LSST will probe the luminosity function to $M_{B}=-23$ at $z \sim 2$, breaking the luminosity-redshift degeneracy that arises in current flux-limited samples such as the Sloan Digital Sky Survey (SDSS), which can only detect $M_{B}>-23$ quasars at $z<0.5$. Also, our understanding of AGN fueling mechanisms and the detailed physics of accretion disks is far from complete (for a review, see McHardy 2010). Therefore, larger and more representative samples will only provide more potential for discovery.

Unfortunately, the resemblance of unresolved quasars (i.e., Type I broad line AGN) to stars in photometric surveys poses a difficulty in their identification. Quasars can be reliably identified by their spectra, and the SDSS provides a spectroscopically complete survey to $i<19$ for the quasar region in SDSS color space (Richards et al. 2002) which includes $\sim 105,000$ quasars (Schneider et al. 2010). However, it is becoming increasingly important to identify quasars in the absence of spectra, particularly since complete spectroscopy will be too expensive in upcoming large-area sky surveys. Previously, quasar selection in the absence of spectroscopy has best been achieved using $u-g$ and $g-r$ colors (e.g., Richards et al. 2002; Jiang et al. 2006; Richards et al. 2009). This technique requires multiple filters and results in samples with efficiencies 1 as low as $73 \%$, and there is a redshift regime near $z \simeq 3$ where optical color selection largely fails. The upcoming large-area sky surveys, such

\footnotetext{
${ }^{1}$ Throughout this paper, "efficiency" $(E)$ is equivalent to "purity" $(p)$ in Schmidt et al. (2010).
} 
as LSST and Pan-STARRS (Kaiser et al. 2002), plan to use their time-domain information to separate quasars from stars. In addition to color selection, it is expected that the lack of proper motion and selection by photometric variability properties will result in fairly clean samples with an efficiency much higher than the color-based 73\%. Also, having an alternative method to color selection is needed to avoid a bias against extincted systems with non-standard colors. However, until very recently, it was not clear how variability could be used in a quantitative fashion to achieve these goals.

A simple way of using variability to separate quasars from stars is to take advantage of the fact that quasars are generally more variable than stars. Sesar et al. (2007) demonstrated that at least $90 \%$ of quasars have a root-mean-square (rms) variability of at least 0.03 mag. However, the rms by itself does not enable the separation of quasars from other variable objects such as RR Lyrae. A recent study by Schmidt et al. (2010, hereafter Schm10) showed that by measuring the rms as a function of time lag $(\Delta t)$ between observations, i.e., the structure function $S F(\Delta t)=A(\Delta t / 1 \mathrm{yr})^{\gamma}$, the parameters $A$ and $\gamma$ enable the separation of quasars and stars with great efficiency $(\approx 95 \%$ for ultraviolet-excess objects). This separation is efficient because the rms of quasar variability is a few times less on monthly than on yearly time scales. Boutsia et al. (2009) showed that the variability of AGN enables their selection even for extended, host-dominated AGN (and X-ray quiet AGN).

An important piece of information missing from these analyses is the characteristic time scale for quasar variability, $\tau$. Studies by de Vries et al. (2005) and Sesar et al. (2006) using SDSS combined with earlier Palomar Observatory Sky Survey measurements for 40,000 SDSS quasars constrained quasar continuum variability on time scales of 10 to 50 years in the observer's frame. They report that the characteristic time scale, which in this context is the time lag above which $S F(\Delta t)$ flattens to a constant value, is of order 1 year 
in the quasar rest frame. Recently, Kelly et al. (2009, hereafter KBS09) proposed a model where the optical variability of a given quasar is described by a damped random walk (DRW). The difference with respect to the well-known random walk is that an additional self-correcting term pushes any deviations back towards the mean flux on a time scale $\tau$. It has been established by KBS09, Kozłowski et al. (2010, hereafter Kozł10), and MacLeod et al. (2010) that a DRW can statistically explain the observed light curves of quasars at an impressive fidelity level (0.01-0.02 mag). The model has only three free parameters: the mean value of the light curve, the driving amplitude of the stochastic process, and the damping (or characteristic) time scale $\tau$. The predictions are only statistical, and the random nature reflects our uncertainty about the details of the physical processes. Kozł10 showed that the DRW model is an efficient quantitative method to separate quasars from most variable stars even in the dense stellar environments of the Magellanic Clouds.

Here, we test quasar selection in extragalactic fields by applying the DRW model to the individual light curves of $\sim 10,000$ variable point sources with $i<19$ from SDSS Stripe 82 (S82). This was also recently done by Butler \& Bloom (2010, hereafter B\&B) using a different approach based on the DRW model. About 15\% of these sources are spectroscopically confirmed quasars whose variability properties have been analyzed in detail by MacLeod et al. (2010). We extend the selection technique in Schm10 by including information on the characteristic (damping) time scale, extend the study of Kozł10 to an extragalactic survey with extensive spectroscopic data, and extend the study in B\&B by applying the DRW model to individual light curves rather than to the ensemble variability of quasars. We describe our variability model in Section 2, describe the S82 data set in Section 3, and present our results in terms of efficiency and completeness in Section 4. In Section 5, we explore the effects that the light curve length, cadence, and photometric errors have on the best-fit DRW parameters. We investigate the performance of the quasar selection technique presented here, in Kozł10, and in B\&B, for typical (simulated) LSST 
and Pan-STARRS cadences. We summarize our findings in Section 6 .

\section{Quasar Variability as a Damped Random Walk}

We model the time variability of quasars as a stochastic process described by the exponential covariance matrix

$$
S_{i j}=\sigma^{2} \exp \left(-\left|t_{i}-t_{j}\right| / \tau\right)
$$

between times $t_{i}$ and $t_{j}$. As detailed by KBS09 and Kozł10, this corresponds to a DRW (more specifically, an Ornstein-Uhlenbeck process) with a damping, or characteristic, time scale $\tau$, and a long-term standard deviation of variability $\sigma$. The driving amplitude of short-term variations is defined as $\hat{\sigma}=\sigma \sqrt{2 / \tau}$. Following Kozł10, we model the light curves and estimate the parameters and their uncertainties using the method of Press et al. (1992), its generalization in Rybicki \& Press (1992), and the fast computational implementation described in Rybicki \& Press (1994). Kozł10 demonstrate that this approach is more statistically powerful than the forecasting methods used by KBS09, while still having computation times scaling linearly with the number of data points.

As in MacLeod et al. (2010), we express the long-term variability in terms of the structure function (SF), where the SF is the rms magnitude difference as a function of the time lag $(\Delta t)$ between measurements. The characteristic time scale for the SF to reach an asymptotic value $\mathrm{SF}_{\infty}$ is the damping time scale, $\tau$. The $\mathrm{SF}$ for a $\mathrm{DRW}$ is

$$
S F(\Delta t)=\mathrm{SF}_{\infty}\left(1-\mathrm{e}^{-|\Delta \mathrm{t}| / \tau}\right)^{1 / 2}
$$

and the asymptotic value at large $\Delta t$ is

$$
S F(\Delta t>>\tau) \equiv \mathrm{SF}_{\infty}=\hat{\sigma} \sqrt{\tau} .
$$


For short time lags,

$$
S F(\Delta t<<\tau)=\mathrm{SF}_{\infty} \sqrt{\frac{|\Delta \mathrm{t}|}{\tau}}=\hat{\sigma} \sqrt{|\Delta \mathrm{t}|} .
$$

Therefore, $\hat{\sigma}$ regulates the rise of $S F(\Delta t<<\tau)$. The $\hat{\sigma}$ parameter is related to $\gamma$ in the equation $S F(\Delta t)=A(\Delta t / 1 \mathrm{yr})^{\gamma}$, where $A$ and $\gamma$ were used to select quasars in Schm10. However, $A$ and $\gamma$ do not provide unique information on the characteristic time scale $\tau$ (although since $\hat{\sigma} \propto \tau^{-1 / 2}$, there is some information on $\tau$ ). While we obtain individual estimates of $\tau$ and $\hat{\sigma}$ for every light curve, in $\mathrm{B} \& \mathrm{~B} \tau$ and $\hat{\sigma}$ are estimated based on the apparent magnitude, which follows from modeling the ensemble SF as a DRW as a function of magnitude. We discuss this difference further in Section 4.2.2.

In Section 4, we demonstrate that the inclusion of $\tau$, in addition to $\hat{\sigma}$, in the selection of quasars enables the selection of highly pure samples with high completeness.

\section{The SDSS Stripe 82 Data Set}

The Sloan Digital Sky Survey (SDSS, York et al. 2000) provides homogeneous and deep $(r<22.5)$ photometry in five passbands (ugriz, Fukugita et al. 1996; Gunn et al. 1998; Smith et al. 2002). To test our quasar selection method, we utilize the $g$-band light curves from the SDSS Stripe 82 (S82), which covers the sky region defined by $22 \mathrm{hr} 24 \mathrm{~m}<\mathrm{RA}<4 \mathrm{hr} 8 \mathrm{~m}$ and $-1.27^{\circ}<$ Dec $<1.27^{\circ}$ (an area of $\left.\approx 290 \mathrm{deg}^{2}\right)$. The light curves span about ten years, and the observations are clustered into yearly seasons about 2-3 months long. There are on average more than 60 available epochs. Because some observations were obtained in non-photometric conditions, improved calibration techniques

have been applied to the SDSS S82 data by Ivezić et al. (2007) and Sesar et al. (2007), and we use their results. For these data, photometric zero-point errors are 0.01-0.02 mag.

We apply the DRW model to all objects in the S82 variable point source catalog (Ivezić 
et al. 2007). These are objects with at least ten observations, an rms of variability in the $g$ and $r$ bands exceeding $0.05 \mathrm{mag}$, and a $\chi^{2}$ per degree of freedom exceeding 3 for the light curves in these bands being fit with a constant flux. We only include observations spaced by at least half a day apart when applying the model, since we are interested in the selection of quasars which generally vary on longer time scales than variable stars. The S82 data set is spectroscopically complete for $i<19$ in the quasar region of $(u-g),(g-r)$, and $(r-i)$ color space (Richards et al. 2002; see the top panel of Figure 1 for the total distribution of quasars in $i$ ). We include newly confirmed DR7 (Abazajian et al. 2009) quasars, and limit our data set to the 52,547 variable objects with $i<19$ when evaluating quasar selection based on variability (Section 4.2). In this sample, there are 1,912 (4\%) spectroscopically identified quasars.

The distribution of the known quasars in RA is compared to the distribution of all variable sources in the bottom panel of Figure 1, The quasar counts fall off at RA $<-35$ deg, while the full distribution rises due to the rising density of Galactic stars. Therefore, we further restrict our sample to the 10,024 objects with $-35^{\circ}<\mathrm{RA}<50^{\circ}$ in order to minimize the stellar contamination from the Galaxy. This sample of 10,024 variable sources, including 1,490 (15\%) spectroscopically confirmed quasars, defines our final sample when evaluating quasar selection based on variability in Section 4.2. However, to demonstrate the performance in regions of increased stellar density, we also compare to the results for the region $-35^{\circ}<\mathrm{RA}<-25^{\circ}$.

It is important to note that for variable objects, the single-epoch $i$ magnitudes can be very different from the best-fit mean magnitudes from the DRW model, $\langle i\rangle$, since the latter are based on the entire time series. In Figure 2, the distribution of $i-\langle i\rangle$ is shown as a function of $\langle i\rangle$ for S82 quasars. It is centered on zero with the $\pm 1 \sigma$ range spanning almost 0.1 mag. Therefore, these differences are significant. For subsequent analysis, we use the 

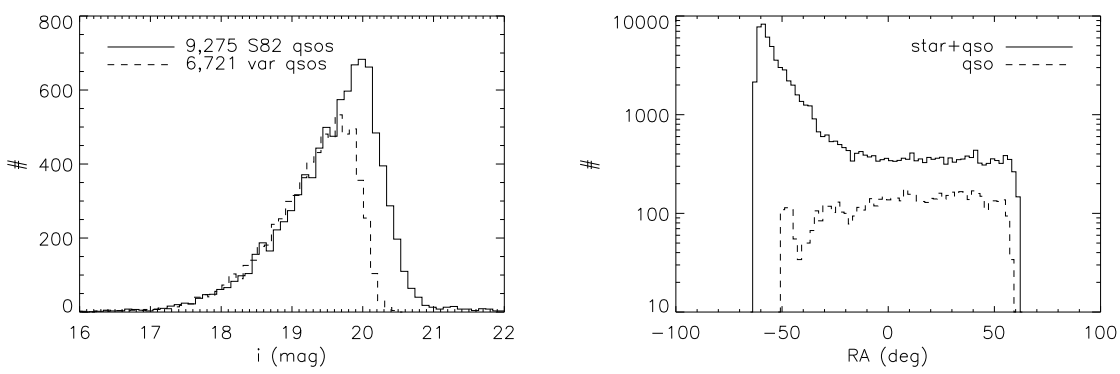

Fig. 1.- Top panel: quasar counts as a function of $i$ magnitude for all spectroscopically confirmed S82 quasars (MacLeod et al. 2010; solid line) and those in the S82 variable point source catalog (Ivezić et al. 2007; dashed line). Bottom panel: counts for all variable point sources (solid) and for spectroscopically confirmed quasars (dashed), as a function of Right Ascension. In order to minimize stellar contamination from the Galactic disk, we only include sources with $-35^{\circ}<\mathrm{RA}<50^{\circ}$ in the subsequent analysis (with Galactic latitudes $\left.-45^{\circ}<b<-37^{\circ}\right)$.

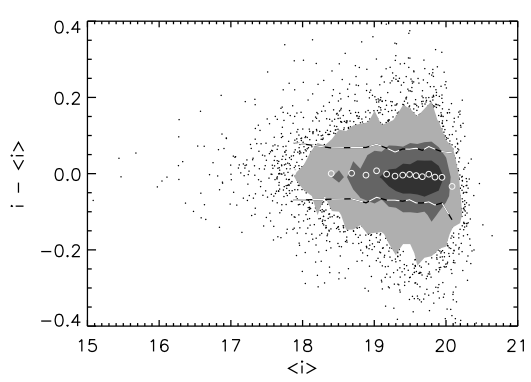

Fig. 2.- Differences in the SDSS BEST $i$ magnitude and best-fit mean $i$ magnitude, $\langle i\rangle$, as a function of $\langle i\rangle$ for S82 quasar light curves modeled as a DRW. The differences arise because the SDSS best $i$ magnitudes are for a single epoch. The $\pm 1 \sigma$ range is shown with dashed lines. The contours show regions containing $25 \%, 50 \%$, and $90 \%$ of the total number of data points.

median PSF $i$-band magnitudes as listed in the variable point source catalog, which are similar to the $\langle i\rangle$ values. All magnitudes are corrected for interstellar dust extinction using Schlegel et al. (1998). 


\section{Results}

\subsection{Variability of Quasars and Other Objects}

In this Section, we present the best-fit variability parameters after applying the DRW model to the light curves of all variable point sources in S82. These parameters are used to develop a method for separating quasars from other variable point sources. We compare the results for two subsamples: one containing the spectroscopically confirmed quasars, and one containing all other objects (which is dominated by stars). We also investigate in detail the objects in the latter subsample that have best-fit time scales greater than 100 days, which is indicative of quasar variability.

Figure 3 shows examples of fitted light curves. The top panel shows a spectroscopically confirmed quasar whose variability is well-fit by the model, and the middle panel shows an unknown object. The weighted average of all model light curves consistent with the data is shown, and the "error snake" is the $\pm 1 \sigma$ range of those light curves about this mean. The best-fit variability parameters along with $\chi_{\mathrm{pdf}}^{2}=\chi^{2} / N_{\mathrm{dof}}$ are listed at the bottom of the panels, where $N_{\text {dof }}$ is the number of degrees of freedom. Additional information on the goodness of fit is provided by the parameter $\Delta L_{\text {noise }} \equiv \ln \left(L_{\text {best }} / L_{\text {noise }}\right)$ which was used in Kozł10 and MacLeod et al. (2010) to select light curves that are better described by a DRW than by pure white noise. Here, $L_{\text {best }}$ is the likelihood of the best-fit stochastic model and $L_{\text {noise }}$ is that for a white noise solution $(\tau \equiv 0)$. In addition to having a larger $\chi_{\text {pdf }}^{2}$, the

light curve for the unclassified object in the middle panel of Figure 3 has $\Delta L_{\text {noise }}=0.4$ and is therefore only marginally more consistent with the DRW model than with white noise. In some cases (see the bottom panel), outliers in the light curve cause a relatively large $\chi_{\mathrm{pdf}}^{2}$ while the likelihood is high for a DRW $\left(\Delta L_{\text {noise }}=34\right)$. Outliers such as these could either represent a model failure or a bad data point with a true error that is larger than the reported value. A detailed analysis of these cases will be discussed in a future publication. 


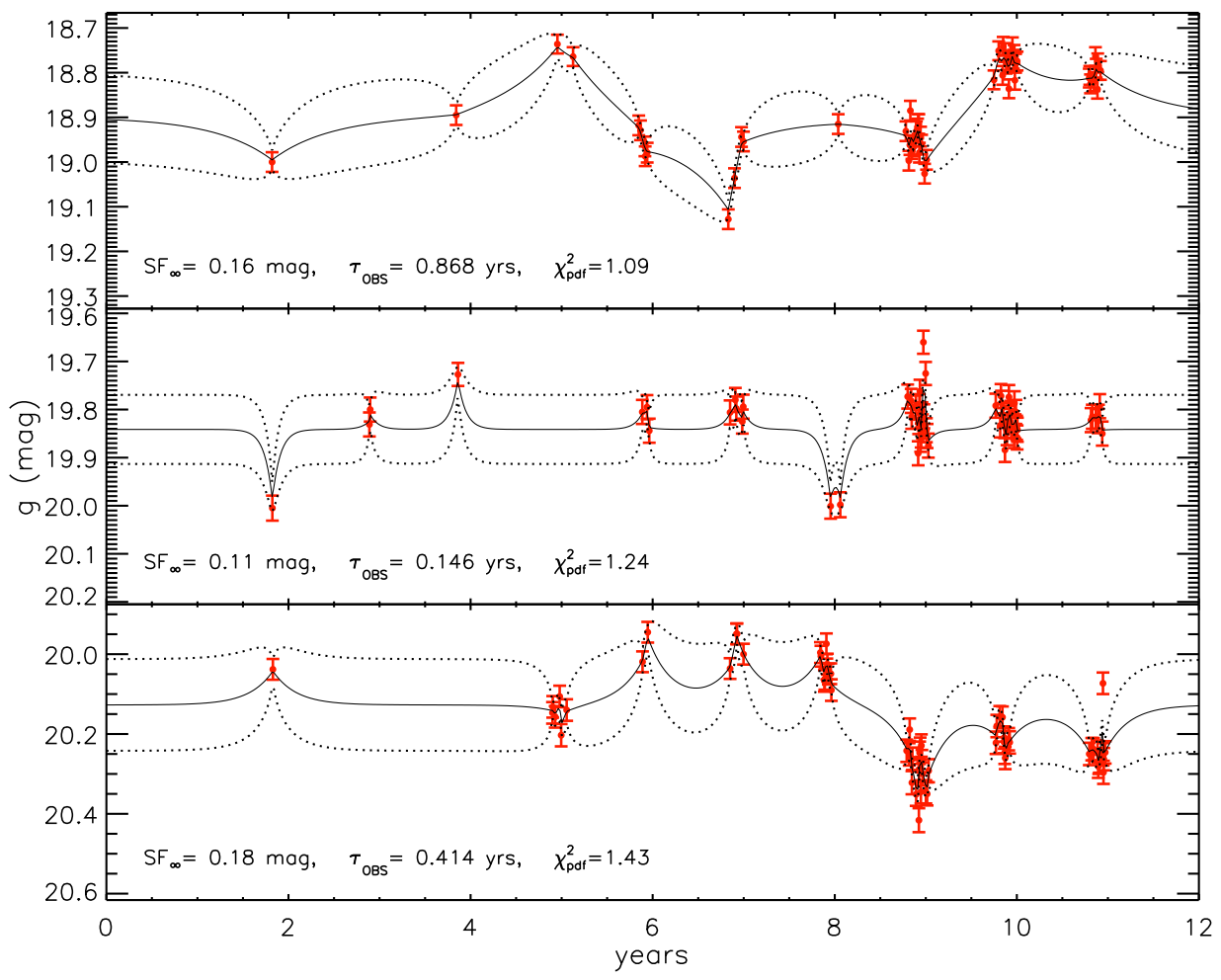

Fig. 3. - The top panel shows a sample light curve for a confirmed quasar. Data points with error bars show the observed data, and the solid line shows the weighted average of all consistent DRW models. Dotted lines show the $\pm 1 \sigma$ range of these possible stochastic models about the average. The middle panel is a similar plot for an unknown object whose light curve can only marginally be distinguished from noise $\left(\Delta L_{\text {noise }}=0.4\right)$. The bottom panel is a similar plot for a confirmed quasar with an outlying data point around 11 years, leading to a large $\chi_{\mathrm{pdf}}^{2}$. The best-fit variability parameters along with $\chi_{\mathrm{pdf}}^{2}=\chi^{2} / N_{\mathrm{dof}}$ are listed at the bottom of the panels.

In Figure 4, $\chi_{\text {pdf }}^{2}$ is shown as a function of $\Delta L_{\text {noise }}$ for the known quasars and all other objects in the variable S82 sample. The quasars typically have $\chi_{\mathrm{pdf}}^{2} \sim 0.85$ while all other variable S82 objects typically have $\chi_{\text {pdf }}^{2} \sim 1.0$, with a large overlap between the two distributions. The overlap is greatly reduced when considering the marginal distributions of $\Delta L_{\text {noise }}$. While $\chi_{\text {pdf }}^{2}$ barely resolves two populations (quasars and stars), $\Delta L_{\text {noise }}$ efficiently separates quasars (which are well-fit by the stochastic model) from variable stars (which are 


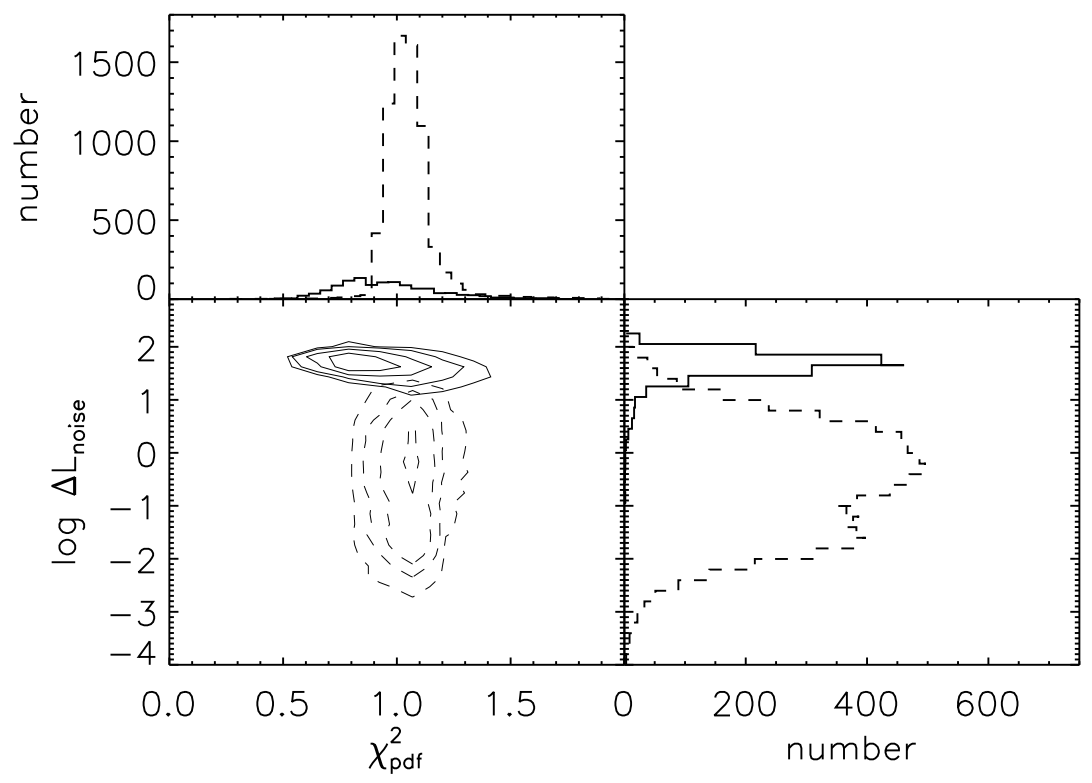

Fig. 4.- Goodness of fit parameters $\chi_{\mathrm{pdf}}^{2}=\chi^{2} / N_{\text {dof }}$ versus $\log \Delta L_{\text {noise }}$ for spectroscopically confirmed quasars (solid) and all other objects (dashed) in our S82 data set restricted to $i<19$. The contours show regions containing $30,60,80$, and $90 \%$ of data points in each subsample. The marginal distributions are shown in the upper and right-hand panels.

better fit by pure noise). Objects with high $\Delta L_{\text {noise }}$ also tend to vary on longer time scales (see Figure 5). Therefore, the characteristic time scale $\tau$ also provides an efficient way to distinguish quasars from stars.

The distributions of $\tau$ and $\mathrm{SF}_{\infty}$ for the quasar and stellar subsamples are shown in Figure 6. The $\tau$ distribution (in the observer's frame) peaks near 500 days for the known quasars and around 1 day for the other objects, providing an efficient and simple way to distinguish between the two subsamples. We note that because quasars are known to have aperiodic variability, eliminating periodic sources should lead to a cleaner sample of quasars. We could classify variable sources as periodic if the likelihood for the peak in the Lomb-Scargle periodogram (as implemented by Press et al. 1992b) being observed at random is $\log \left(p_{\text {periodic }}\right)<-3$ and the estimated period is less than $\sim 200$ days. For longer 


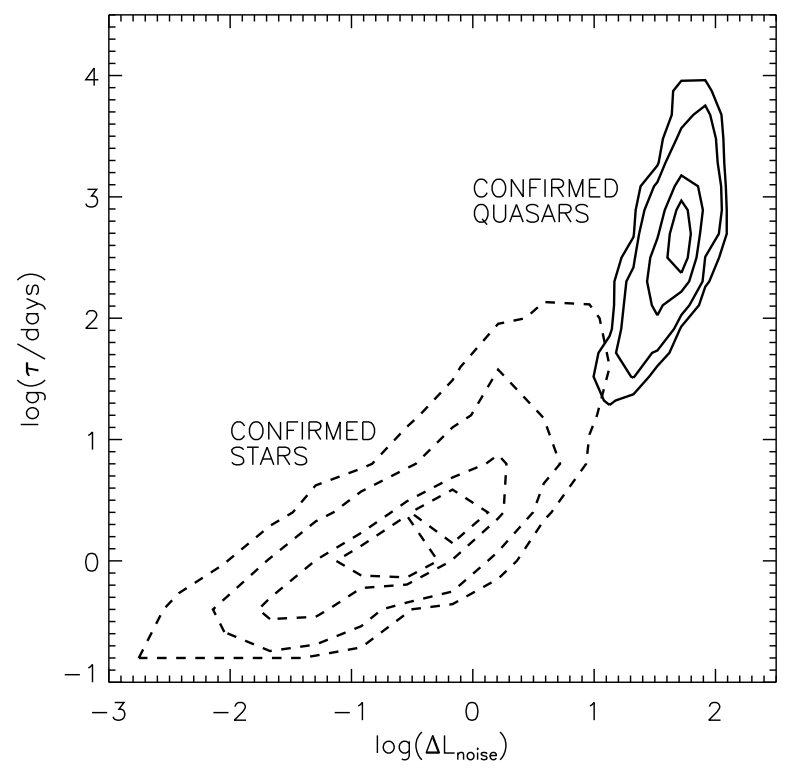

Fig. 5.- Dependence of the best-fit characteristic time scale on the relative likelihood $\Delta L_{\text {noise. Solid }}$ (dashed) contours show the $25 \%, 50 \%, 75 \%$, and $90 \%$ levels for spectroscopically confirmed quasars (stars).

periods, many quasars are falsely flagged as periodic because the periodogram only tests for whether a sine wave is a better fit than a constant, and this is frequently true for quasars with only a few "oscillations" in the data (see Kozł10). Because we have few longer period variable stars in S82, adding such a cut for periodic stars adds little and so we have not included it. This differs from the Magellanic Cloud fields examined by Kozł10 where there are many longer-period periodic stars (e.g., Cepheids).

The use of $\tau$ as an efficient classifier is further illustrated in Figure 7 , The top-left panel shows the $(u-g)$ and $(g-r)$ colors for spectroscopically confirmed quasars and stars. The top-right panel shows the median value of $\tau$ as a function of $(u-g)$ and $(g-r)$ color. This panel shows that the quasar locus (region II) as well as the high redshift quasars $(z \gtrsim 3.2$; region IV) are dominated by long time scales, while the stellar locus (region I) and the RR Lyrae (region III) have short time scales. The time scale information is able to clearly distinguish between quasars and RR Lyrae, as opposed to when using the rms of variability 


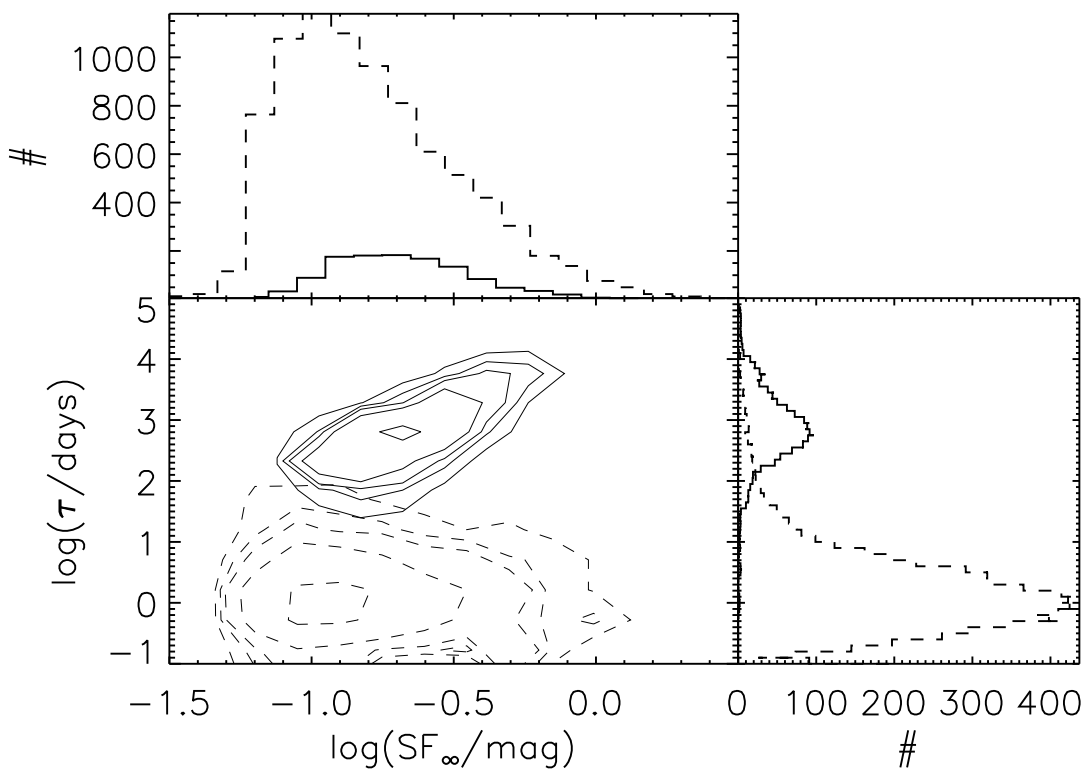

Fig. 6.- Characteristic time scale $\tau$ versus asymptotic $\mathrm{rms}$ variability $\mathrm{SF}_{\infty}$ for spectroscopically confirmed quasars (solid) and all other objects (dashed) in our S82 data set restricted to $i<19$. The contours show regions containing $10,60,75,85$, and $90 \%$ of data points in each subsample. The marginal distributions are shown in the upper and right-hand panels.

alone (see Figure 3 in Sesar et al. 2007). The quasar regions of color space also tend to have larger median values of $\mathrm{SF}_{\infty}$, as seen in the bottom-left panel of Figure 7 . The remaining panel shows the median driving amplitude of short-term variations, $\hat{\sigma}=\mathrm{SF}_{\infty} / \sqrt{\tau}$, against $(u-g)$ and $(g-r)$ color. Because it is anti-correlated with $\tau$ (see Kozł10), the quasar regions show systematically smaller $\hat{\sigma}$ than the stellar regions.

Figure 8 compares the distribution of objects with $\tau \geq 100$ days and $\tau<100$ days in $(u-g, g-r)$ space, as well as the subset of 233 objects with $100 \leq \tau<10^{5}$ days and $\Delta L_{\text {noise }}>2$ that are not spectroscopically confirmed quasars. The latter contains good quasar candidates, especially those in regions II or IV (51 objects). Thirty-five of these are spectroscopically confirmed stars, represented by the star symbols. Figure 9 shows the distribution of these 233 objects in $\left(\tau, \mathrm{SF}_{\infty}\right),(i, i-z)$, and $(r-i, i-z)$ space. The subset in 
regions II or IV, represented by triangles, are clustered near the quasar region at faint $i$ and blue $(r-i, i-z)$ colors. We inspected their observed and phased light curves (using the Supersmoother algorithm, see Reimann 1994), and they appear consistent with quasar light curves with no signs of periodicity. About half of these 51 objects are detected in GALEX2 GR6 with GALEX-SDSS UV-optical colors resembling those of quasars $(n-u<1.5$ and $u-g<0.8$, or $f-n>0$ and $g-r<0.5$; see Agüeros et al. 2005). About $15 \%$ have 2MASS 3 colors $J-K>1$ that are also indicative quasar nature. We also investigated the 15 unknown objects with $\tau \geq 100$ days that lie in region I but off the stellar locus (above the solid line in Figure 8). These objects (represented by asterisks in Figure 96) seem consistent with reddened quasars, as their light curves are consistent with quasars, and they are rather faint and clustered at redder $(r-i, i-z)$ colors. However, their median $\mathrm{SF}_{\infty}(0.15 \mathrm{mag})$ is lower than that for the quasar candidates represented by triangles (0.22 mag). None of these objects have GALEX-SDSS UV-optical colors indicative of quasars, but about 30\% have $J-K>1$. Follow-up spectroscopy will be useful for classifying these two subsamples.

The remaining objects with $\tau \geq 100$ days lying on the stellar locus or in region III seem consistent with stars based on their $i$ magnitude and colors, and many are spectroscopically confirmed as stars. The majority have small amplitudes $\left(\mathrm{SF}_{\infty} \approx 0.1 \mathrm{mag}\right)$. However, there is a subset with large amplitudes $\left(\mathrm{SF}_{\infty}>0.8 \mathrm{mag}\right)$. The majority of these sources have a single outlier at a significantly fainter magnitude in their light curves (e.g., SDSS J001014.28+005938.6, see Figure 10) causing the model to fit a long time scale. These could be wide eclipsing binaries or simply a bad data point. If we reject the data point with the largest magnitude difference from the median in each light curve with $\mathrm{SF}_{\infty}>0.8$ mag,

\footnotetext{
${ }^{2}$ Galaxy Evolution Explorer; see http://galex.stsci.edu/GR6/. A matching radius of 3" was used.

${ }^{3}$ Two Micron All Sky Survey; see http://www.ipac.caltech.edu/2mass/. A matching radius of 1.5" was used.
} 
and then refit the models, half now have $\mathrm{SF}_{\infty}<0.2$ mag and $60 \%$ now have $\Delta L_{\text {noise }}<2$ and $\tau<100$ days. There is a subset of these sources with large amplitude semi-periodic outbursts, suggestive of AGB stars (e.g., SDSS J220514.58+000845.7, see Figure 10). In general, however, inspection of both the phased and observed light curves revealed no examples of truly periodic behavior. A few $(2 \%)$ of these objects have GALEX-SDSS UV-optical colors indicative of quasars, and about $5 \%$ have $J-K>1$.

We compile a list of quasar candidates by removing those that already have SDSS spectra from the list of 233 and including cases where $\tau$ has saturated to $10^{5}$ days. We retain 255 objects lacking SDSS spectra that have $i<19, \Delta L_{\text {noise }}>2$, and $\tau \geq 100$ days, leading to a sample of 255. The coordinates, SDSS photometry, and DRW parameters for each target are provided in Table 1. These represent our target sample for spectroscopic follow-up. Note that $30 \%$ of the target sample fails the $\Delta L_{\text {noise }}>2$ and $\tau \geq 100$ day criteria when omitting the most outlying data point in the light curve. 

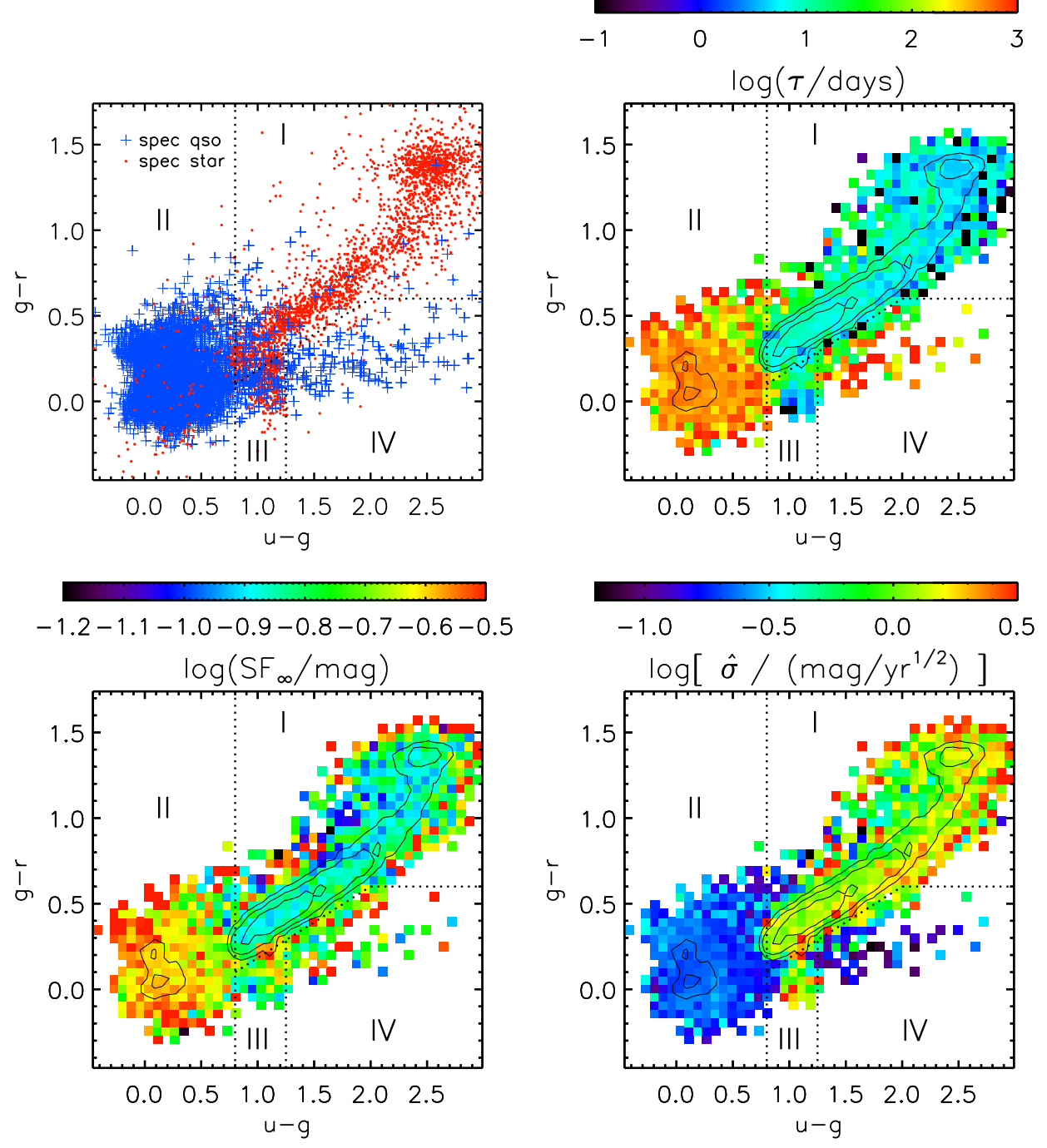

Fig. 7.- Top-left: $u-g$ versus $g-r$ colors of all spectroscopically confirmed S82 quasars (crosses) and spectroscopically confirmed stars (dots). Top-right: The median characteristic time scale $\tau$ for all variable point sources in S82 that are well-described by the DRW model $\left(\Delta L_{\text {noise }}>2\right)$, as a function of $u-g$ and $g-r$, following the legend at the top. The contours show regions containing $25 \%, 50 \%$, and $75 \%$ of the total number of data points. The red regions with large $\tau$ coincide with the quasar color regions, providing a convenient method for quasar selection based on variability. Bottom-left: As in top-left panel but for the asymptotic rms of variability, $\mathrm{SF}_{\infty}$. Bottom-right: As in top-left panel but for the driving amplitude of short-term variations, $\hat{\sigma}=\mathrm{SF}_{\infty} / \sqrt{\tau}$. The dotted lines divide the color space into four characteristic regions: the stellar locus (I), the low-redshift $(z \lesssim 2)$ quasar region (II), the RR Lyrae region (III), and the high-redshift $(z \gtrsim 3.2)$ quasar region (IV). 


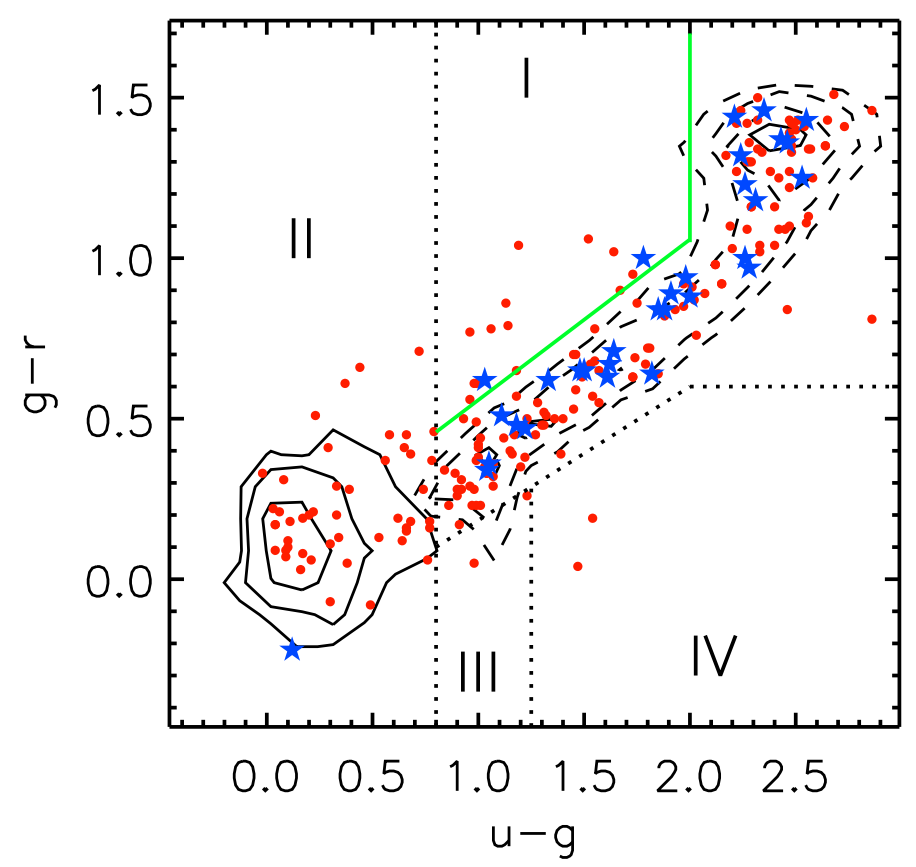

Fig. 8. - $u-g$ versus $g-r$ colors of bright $(i<19)$ variable point sources with $\tau \geq 100$ days (solid contours) and $\tau<100$ days (dashed; 25\%, 60\%, and $75 \%$ levels). Also shown are the 233 objects with $100 \leq \tau<10^{5}$ days and $\Delta L_{\text {noise }}>2$ that are not spectroscopically confirmed quasars (red dots). Those with confirmed stellar origin are represented by blue stars. We use the solid line in region I to define a subsample of objects that lie off the stellar locus. 

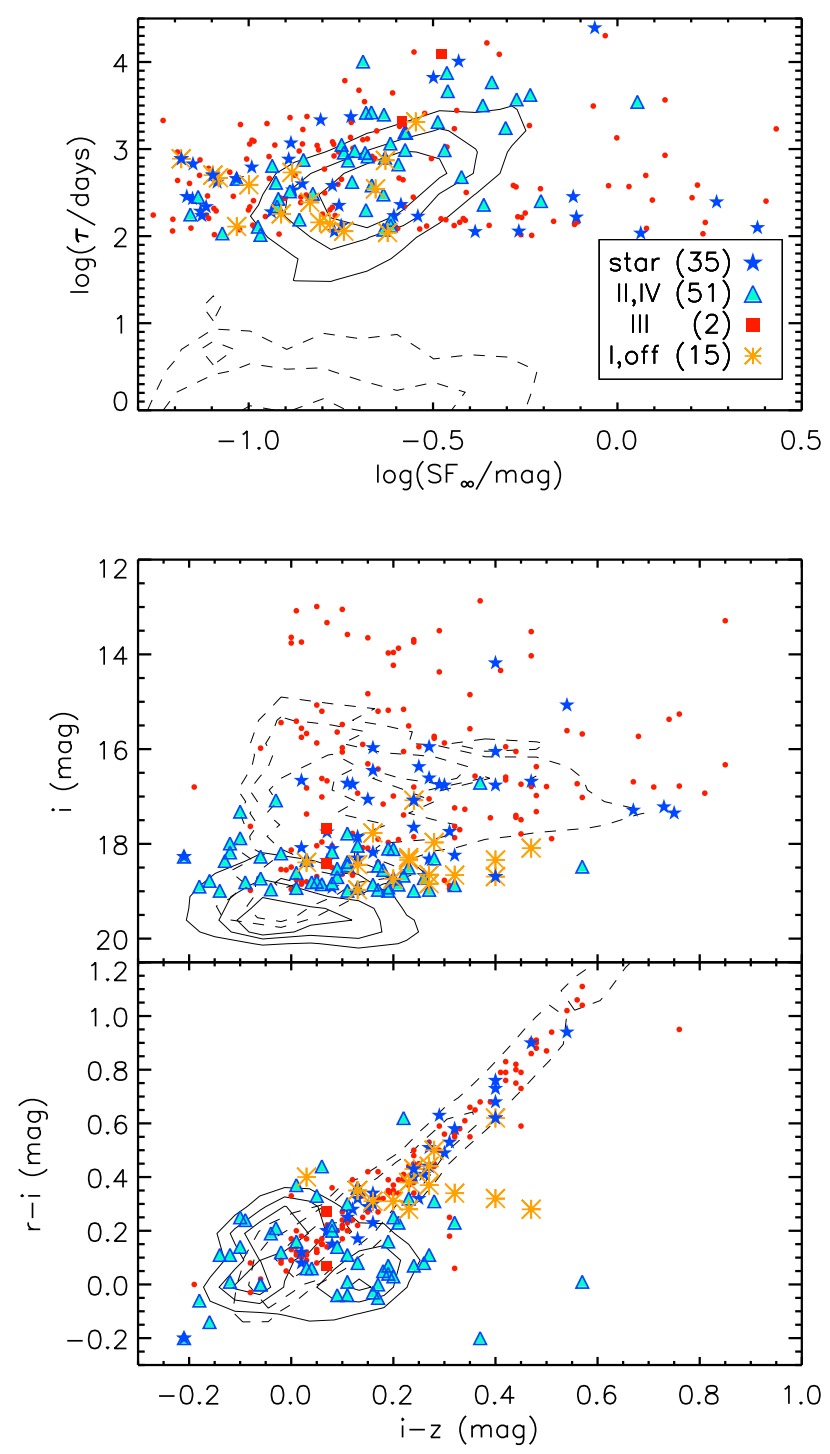

Fig. 9. - Top: Variability time scale $\tau$ versus amplitude $\mathrm{SF}_{\infty}$ for all spectroscopically confirmed quasars (solid contours), all confirmed stars (dashed), and the 233 objects with $100 \leq \tau<10^{5}$ days, $\Delta L_{\text {noise }}>2$, and $i<19$ that are not already confirmed quasars (symbols). Blue stars represent those with confirmed stellar origin, and cyan triangles represent those with quasar-like colors (regions II or IV of Figure 7). Red squares represent those in region III, and orange asterisks represent those that lie in region I but above the solid line in Figure 8. The number of objects in each subset is listed in the legend, and the remaining 130 objects are represented by red dots. The contour levels are 25\%,50\%, and 75\%. Middle and bottom: As in top panel but for $i$ magnitude and $r-i$ color, respectively, as a function of $i-z$. 


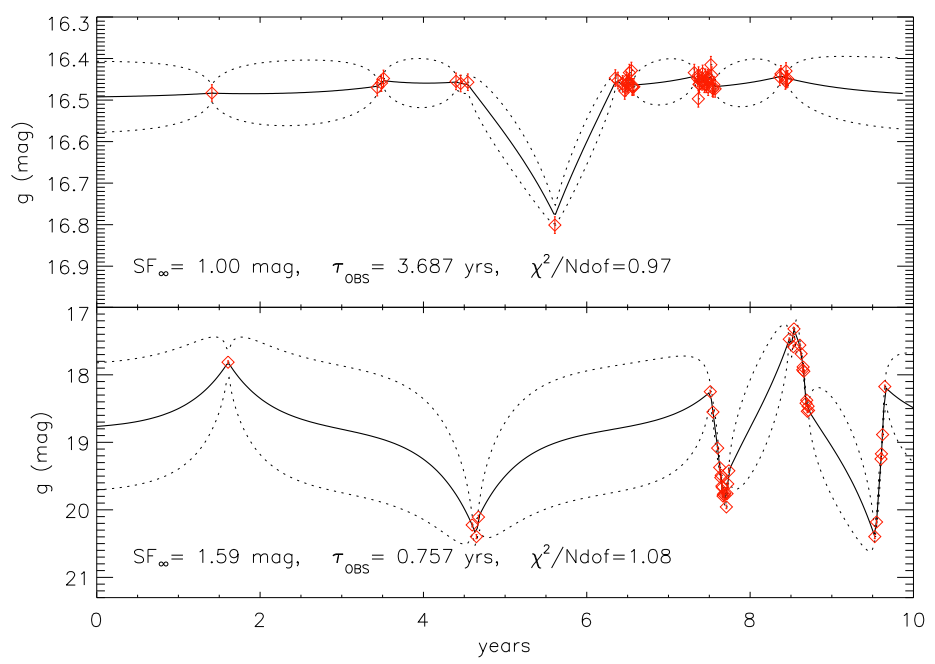

Fig. 10. - Light curves for SDSS J001014.28+005938.6, a probable example of a false variable created by a bad data point (top), and SDSS J220514.58+000845.7, a probable example of a non-periodic variable star (bottom). Data points with error bars show the observed data, and the solid line shows the weighted average of all consistent DRW models. Dotted lines show the $\pm 1 \sigma$ range of these possible stochastic models about the average. The best-fit variability parameters are listed at the bottom of the panels.

Table 1. Targets for Spectroscopic Follow-up

\begin{tabular}{crrcccccccc}
\hline \hline ID & RA (deg) & Dec (deg) & $i$ & $u-g$ & $g-r$ & $r-i$ & $i-z$ & $\log (\tau /$ days $)$ & $\mathrm{SF}_{\infty}(\operatorname{mag})$ & Outlier \\
\hline & & & & & & & & & & \\
7912248 & 340.000458 & 0.304811 & 15.73 & 2.32 & 1.43 & 1.24 & 0.68 & 2.34 & 0.26 & 1 \\
46516 & 3.922421 & -0.331890 & 17.97 & 2.02 & 0.87 & 0.35 & 0.19 & 2.06 & 0.06 & 0 \\
48527 & 2.699158 & -0.234567 & 13.67 & 1.95 & 0.70 & 0.22 & 0.11 & 5.00 & 1.39 & 1 \\
\hline \hline
\end{tabular}

Note. - Stripe 82 object ID, J2000 coordinates, SDSS photometry, and DRW parameters for 255 unknown objects with $\tau \geq 100$ days, $\Delta L_{\text {noise }}>2$, and $i<19$. For some objects, the time scale has saturated to $\tau=10^{5}$ days due to insufficient light curve lengths. "Outlier" is set to 1 for objects that fail the $\tau \geq 100$ day and $\Delta L_{\text {noise }}>2$ selection criteria after rejecting the data point furthest from the median magnitude. (See the online version for the complete table.) 


\subsection{Completeness and Efficiency}

To measure the success of our quasar selection, we report the completeness $(C)$ and efficiency $(E)$, defined by

$$
\begin{aligned}
& C=\frac{\# \text { of selected confirmed quasars }}{\text { total \# of confirmed quasars }} \times 100 \\
& E=\frac{\# \text { of selected confirmed quasars }}{\text { total \# of selected objects }} \times 100
\end{aligned}
$$

for a variety of selection criteria, where "confirmed" means that the source is spectroscopically identified as a quasar. Here, we only consider sources with $i<19$, and $E$ is a lower limit for the true efficiency because the sample of confirmed quasars may not be $100 \%$ spectroscopically complete. These quantities for a quasar selection based on $\tau$ alone are shown in Figure 11 as a function of the minimum threshold $\tau$. The two quantities are anti-correlated due to the increased overall fraction of selected quasars as well as the increased number of missed quasars when restricting $\tau$ to longer time scales. The completeness drops to $94 \%$ (where $E=81 \%$ ) at a threshold of $\tau>100$ days, beyond which $C$ dramatically decreases. At the same time, $E$ for $\tau>100$ days is almost as high as its asymptotic value (85\%). Therefore, we adopt $\tau \geq 100$ days as the optimal cut for selecting quasars based on time scale alone. An additional constraint of $\Delta L_{\text {noise }}>2$ (thick line in Figure 11) does not affect the resulting $C$ or $E$ by more than $1 \%$ after requiring $\tau \geq 100$ days, while the constraint $\Delta L_{\text {noise }}>10$ (gray lines) increases $E$ with a minor drop in $C$.

Table 2 lists $C$ and $E$ for various selection criteria, beginning with the $i$ magnitude and RA limits that define our starting sample (first row, hence the $100 \%$ completeness), and the

restrictions $\tau \geq 1$ and 100 days (second and third rows). When excluding the objects that fail the $\Delta L_{\text {noise }}>2$ and $\tau \geq 100$ day criteria after omitting the most outlying data point in the light curve, $E$ rises from $81 \%$ to $87 \%$, while $C$ only drops by $1 \%$ (fourth row). This cut 


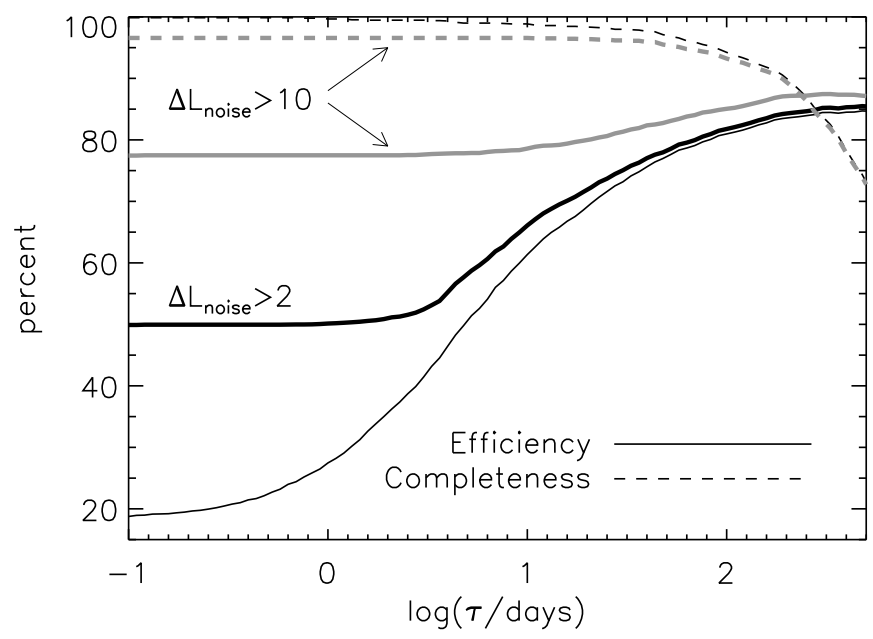

Fig. 11. - E (solid curves) and $C$ (dashed curves) measured as a function of the minimum threshold $\tau$ for variable S82 point sources with $i<19$ and $-35^{\circ}<\mathrm{RA}<50^{\circ}$. The thin, solid and dashed black curves show the results when using a $\tau$ criterion alone. The thick, solid black curve shows the effect of adding a criterion of $\Delta L_{\text {noise }}>2(C$ is unaffected $)$, and the gray curves show the effect of adding $\Delta L_{\text {noise }}>10$.

is useful for eliminating potentially bad data in order to boost the efficiency of the selected sample. $C$ can be increased to $96 \%$ while maintaining $E=87 \%$ by reducing the limit to $\tau \geq 10^{1.5}$ days and requiring $\Delta L_{\text {noise }}>10$ (fifth row). Here, we have excluded objects that drop out of the selection after removing the most outlying data point. When further restricting the variability-selected sample (with $\tau \geq 100$ days) to those objects which have quasar colors (regions II and IV in Figure 7), $C$ decreases to $91 \%$ while $E$ rises to $96 \%$ (sixth row; these results are unaffected when rejecting light curve outliers). Table 2 also lists the percentage of each selected sample that have SDSS spectra, and the breakdown into quasars, stars, and galaxies based on their SDSS spectroscopic class.

We also compute $E$ and $C$ for the ultraviolet-excess ("UVX") and non-ultraviolet-excess ("nUVX") color boxes as defined in Schm10. The former should be spectroscopically complete to $i<19$ (see Richards et al. 2002). The latter corresponds to the quasar redshift 
regime of $\sim 3$, where color selection largely fails. Here, $C$ is the percentage of all confirmed variable (n)UVX quasars that have $\tau \geq 100$ days. For UVX objects, $C=95 \%$ and $E=97 \%$ for the $\tau \geq 100$ days selection criteria (eighth row of Table 2). For comparison, the Schm10 selection method, which does not use the $\tau$ information, gives $C=90 \%$ and $E \simeq 95 \%$. For nUVX objects (only 52 objects; tenth row), $C=100 \%$ for the $\tau \geq 100$ days selection criteria, and $E$ is only $69 \%$ due to the fact that only $69 \%$ have spectra.

The driving amplitude $\hat{\sigma}$ also shows a large contrast between the quasar color regions (II and IV) and the stellar regions (I and III) in Figure 7 . This suggests that including information on the driving amplitude may improve the quasar selection based on variability. Indeed, Schm10 found high-efficiency quasar samples by using information closely corresponding to $\hat{\sigma}$. In Figure 12, we show how the selection can be improved by including information on both $\tau$ and $\hat{\sigma}$. In the top two panels, $E$ and $C$ are mapped out as a function of the minimum $\tau$ and the maximum $\hat{\sigma}$ allowed for quasar classification. For every value of $\hat{\sigma}$, there is a lower limit on $\tau$ that boosts efficiency with a minor drop in $C$. This essentially follows one of the quasar selection criteria for $\tau$ and $\hat{\sigma}$ in Kozł10. A similar improvement is seen when instead using lower limits in both $\tau$ and $\mathrm{SF}_{\infty}$, or a combination of $\tau, \mathrm{SF}_{\infty}$, and $\hat{\sigma}$ information (the differences are all $\lesssim 1 \%$ ). In the bottom panels of Figure 12, it can be seen more quantitatively how the inclusion of $\tau$ improves the selection of quasars as compared to using $\hat{\sigma}$ alone. The panels show the maximum $C$ achieved for an $E$ exceeding that plotted on the x-axis, for three cases. In the first case (solid), only the $\hat{\sigma}$ parameter is used to separate quasars and stars, i.e., all values of $\tau$ are allowed, as in Schm10. The second case (dashed) shows the effect of including information on $\tau$. The third case (dotted) shows results for using $\tau$ information alone. When including all SDSS colors (bottom-left panel), the $\tau$ information alone leads to significantly higher $E$ than a selection based on $\hat{\sigma}$ alone. However, for UVX objects (bottom-right panel), a combination of $\tau$ and $\hat{\sigma}$ information is required to significantly boost $E$ from a selection based on $\hat{\sigma}$ 
alone.

Using both $\tau$ and $\hat{\sigma}$ as criteria for quasar selection in the absence of color information, one can achieve an efficiency of $E=82 \%$ with a completeness of $C=96 \%$ (seventh row of Table 21), or alternatively, $E=75 \%$ for $C=98 \%$, and $E=85 \%$ for $C=90 \%$. Here, objects that drop out of the selection after omitting the most outlying data point in the light curve are excluded. When restricting the selection to color regions II and IV in Figure 7, one can achieve $E=96 \%$ with $C=93 \%$. At the same level of completeness, $E=84 \%$ without color cuts, and $E=78 \%$ when using $\hat{\sigma}$ alone. For UVX objects, $E=97 \%$ and $C=98 \%$ when

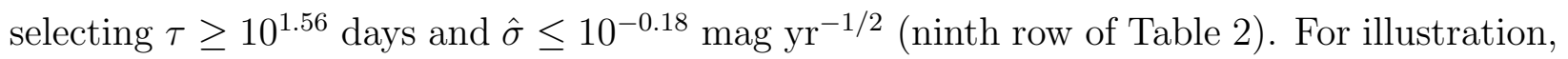
Figure 13 shows $\tau$ versus $\mathrm{SF}_{\infty}$ for the variable $\mathrm{S} 82$ sample, with lines of constant $\hat{\sigma}$ overlaid. By choosing $\hat{\sigma}<10^{-0.2} \mathrm{mag} \mathrm{yr}^{-1 / 2}$, the entire quasar locus is selected as well as the gray shaded region containing stars. However, when imposing a lower limit at $\tau=10^{1.56}$ days, these contaminants are excluded from the sample, leading to a higher efficiency of quasar selection.

Since the selection of quasars based on variability is a useful tool in regions of high stellar density (Kozł10), we repeat the analysis in Table 2 for the region $-35^{\circ}<\mathrm{RA}<-25^{\circ}$, where the density of Galactic stars rises. Since the rate of contamination is higher in this region, it is useful to reject outliers in light curves in order to boost efficiency as in the fourth row of Table 2. These results can be found in Table 3. Here, $E$ for the $\tau \geq 100$ days selection is $79 \%$, after omitting outliers in light curves. Restricting this selection to $\Delta L_{\text {noise }}>10$ boosts $E$ to $82 \%$ without a drop in completeness. Therefore, a strict cut in $\Delta L_{\text {noise }}$ is especially useful in regions of high stellar density. 
Table 2. Selection Criteria for $-35^{\circ}<\mathrm{RA}<50^{\circ}$ and $i<19$

\begin{tabular}{|c|c|c|c|c|c|c|c|c|c|c|c|}
\hline & Selection & $N$ & $C$ & $\delta C$ & $E$ & $\delta E$ & Spec. & QSO & Star & Gal. & Unk. \\
\hline 1. & All Sources & 10024 & 100 & 4 & 15 & 0.4 & 33 & 45 & 53 & 2.5 & 0.15 \\
\hline 2. & $\tau>1 \mathrm{~d}$ & 5385 & 99.7 & 4 & 28 & 0.8 & 43 & 64 & 33 & 2.0 & 0.22 \\
\hline 3. & $\tau \geq 100 \mathrm{~d}$ & 1734 & 94 & 3 & 81 & 3 & 84 & 96 & 3.5 & 0.55 & 0.14 \\
\hline 4. & $\tau \geq 100 \mathrm{~d}^{*}$ & 1594 & 93 & 3 & 87 & 3 & 89 & 98 & 1.76 & 0.49 & 0.14 \\
\hline 5 & $\tau \geq 10^{1.5} \mathrm{~d}, \Delta L_{\text {noise }}>10^{*}$ & 1647 & 96 & 4 & 87 & 3 & 89 & 97 & 2.0 & 0.61 & 0.20 \\
\hline 6. & $\tau \geq 100 \mathrm{~d}$, qso-like colors & 1420 & 91 & 3 & 96 & 4 & 97 & 99 & 0.15 & 0.44 & 0.15 \\
\hline 7. & $\tau \geq 10^{1.56} \mathrm{~d}, \hat{\sigma} \leq 10^{-0.18} \mathrm{mag} \mathrm{yr}^{-1 / 2 *}$ & 1751 & 96 & 4 & 82 & 3 & 86 & 96 & 3.3 & 0.87 & 0.07 \\
\hline 8. & $\tau \geq 100 \mathrm{~d}$ & 1297 & 95 & 3 & 97 & 4 & 98 & 99 & 0.16 & 0.32 & 0.16 \\
\hline 9. & $\tau \geq 10^{1.56} \mathrm{~d}, \hat{\sigma} \leq 10^{-0.18}{\operatorname{mag~} \mathrm{yr}^{-1 / 2}}^{-1}$ & 1332 & 98 & 3 & 97 & 4 & 98 & 99 & 0.15 & 0.31 & 0.08 \\
\hline 10. & $\tau \geq 100 \mathrm{~d}$ & 52 & 100 & 0.4 & 69 & 15 & 69 & 100 & 0.00 & 0.00 & 0.00 \\
\hline 11. & $B \& B$ & 1702 & 96 & 4 & 84 & 3 & 87 & 97 & 2.7 & 0.40 & 0.13 \\
\hline
\end{tabular}

*The most outlying data point in each light curve is omitted.

Note. - The first column lists various selection criteria ("qso-like colors" signifies having $u-g$ and $g-r$ colors in regions II or IV of Figure 7). $N$ is the total number of objects that satisfy each criterion (the sample is defined by $i<19$ and covers $213 \mathrm{deg}^{2}$ ). $C$ is the quasar completeness, or the percentage of all confirmed quasars in the sample of 10,024 that are selected. $E$ is the efficiency, or the percentage of the selected objects that are confirmed quasars. $\delta C$ and $\delta E$ are the Poisson errors on $C$ and $E$. "Spec." indicates the percentage that have an SDSS spectrum, and the last four columns show the percentage breakdown of the subset with spectra into four spectroscopic classes: QSO (SpecClass is 3 or 4), Star (SpecClass is 1 or 6), Galaxy (SpecClass is 2), and Unknown (SpecClass is 0). In the eighth and ninth rows (tenth row), we list results for the (n)UVX color box described in Schm10, where $C$ is computed as the percentage of all confirmed quasars contained in the (n)UVX color box that satisfy the variability criteria listed in the first column. The eleventh row lists the results for the selection criteria in B\&B (see Section 4.2.2). 
Table 3. Selection Criteria for $-35^{\circ}<\mathrm{RA}<-25^{\circ}$ and $i<19$

\begin{tabular}{|c|c|c|c|c|c|c|c|c|c|c|c|}
\hline & Selection & $N$ & $C$ & $\delta C$ & $E$ & $\delta E$ & Spec. & QSO & Star & Gal. & Unk. \\
\hline 1. & All Sources & 2298 & 100 & 12 & 6.5 & 0.5 & 20 & 32 & 65 & 2.17 & 0.22 \\
\hline 2. & $\tau>1 \mathrm{~d}$ & 991 & 99 & 12 & 15 & 1 & 30 & 50 & 47 & 2.72 & 0.34 \\
\hline 3. & $\tau \geq 100 \mathrm{~d}^{*}$ & 167 & 89 & 11 & 79 & 9 & 83 & 95 & 2.2 & 2.16 & 0.72 \\
\hline 4. & $\tau \geq 100 \mathrm{~d}, \Delta L_{\text {noise }}>10^{*}$ & 160 & 89 & 11 & 82 & 10 & 85 & 97 & 0.74 & 1.47 & 0.74 \\
\hline 5. & $\tau \geq 100 \mathrm{~d}$, qso-like colors ${ }^{*}$ & 137 & 86 & 10 & 93 & 11 & 96 & 98 & 0.76 & 1.53 & 0.76 \\
\hline 6. & $\tau \geq 10^{1.56} \mathrm{~d}, \hat{\sigma} \leq 10^{-0.18} \operatorname{mag} \mathrm{yr}^{-1 / 2}$ & 179 & 93 & 11 & 78 & 9 & 80 & 97 & 1.4 & 1.4 & 0.70 \\
\hline & $\Delta L_{\text {noise }}>10^{*}$ & & & & & & & & & & \\
\hline 7. & $\tau \geq 100 \mathrm{~d}$ & 127 & 89 & 10 & 94 & 12 & 97 & 98 & 0.00 & 1.63 & 0.81 \\
\hline 8. & $\tau \geq 10^{1.56} \mathrm{~d}, \hat{\sigma} \leq 10^{-0.18} \operatorname{mag} \mathrm{yr}^{-1 / 2}$ & 134 & 95 & 10 & 96 & 12 & 97 & 98 & 0.00 & 1.54 & 0.00 \\
\hline 9. & $\tau \geq 100 \mathrm{~d}$ & 10 & 100 & 2 & 50 & 27 & 50 & 100 & 0.00 & 0.00 & 0.00 \\
\hline
\end{tabular}

*The most outlying data point in each light curve has been omitted.

Note. - As in Table 2 but for regions of increased stellar density. 

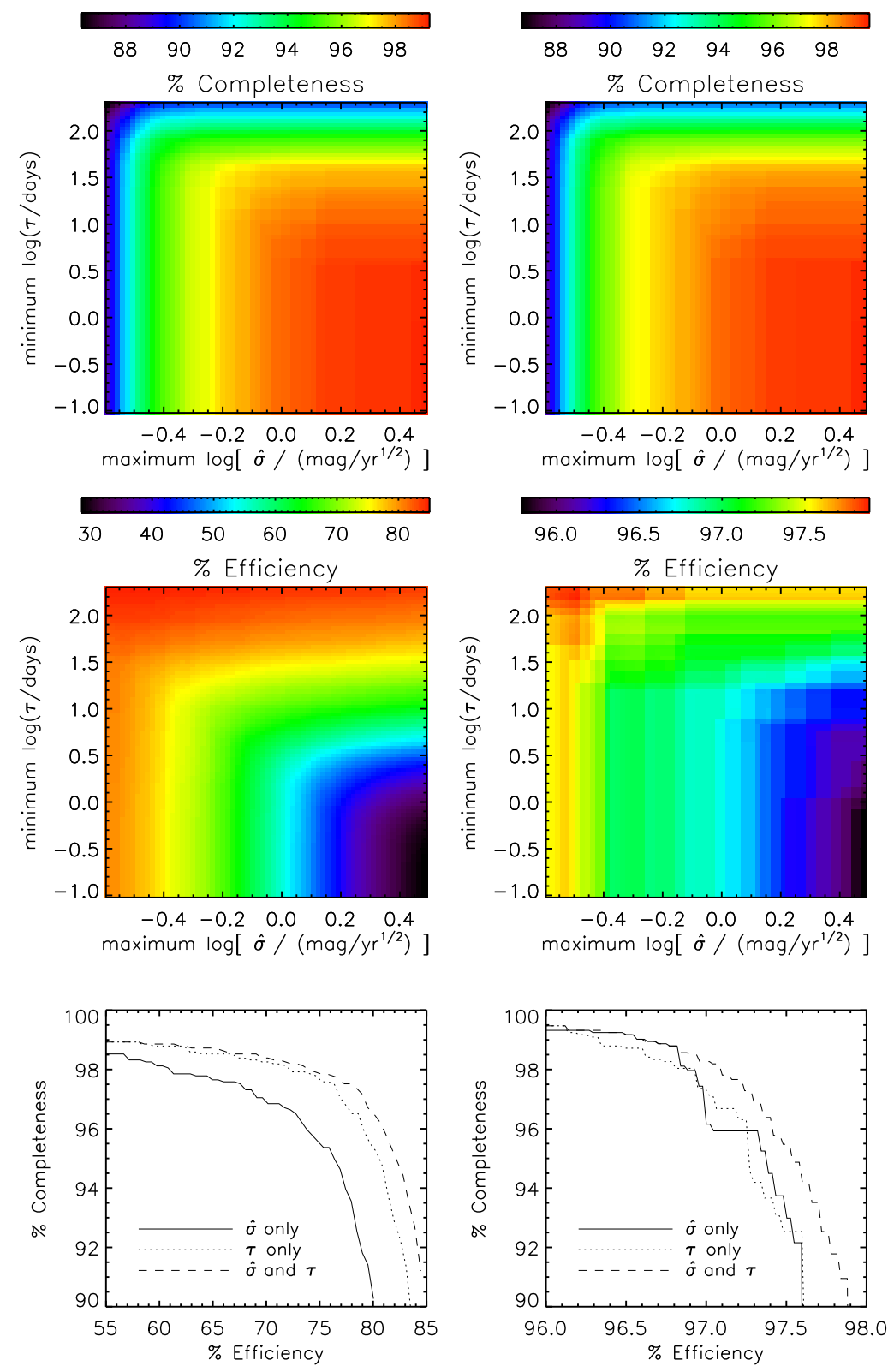

Fig. 12. - Left panels: Improvements in variability-based selection of quasars due to added time scale information. The top two panels show the the completeness $(C)$ and efficiency $(E)$ for quasar classification as a function of the minimum $\tau$ and maximum $\hat{\sigma}$ allowed for the variable sample with $i<19$ and $-35^{\circ}<\mathrm{RA}<50^{\circ}$. The solid line in the bottom panel shows the maximum $C$ as a function of $E$ achieved when using $\hat{\sigma}$ information alone (i.e., allowing all time scales $\tau$ ). The dashed line shows how $E$ improves when including a cut in $\tau$ to select quasars in addition to a cut in $\hat{\sigma}$. The dotted line shows results when using the $\tau$ information alone. Right panels: As in left panels but for UVX objects only. 

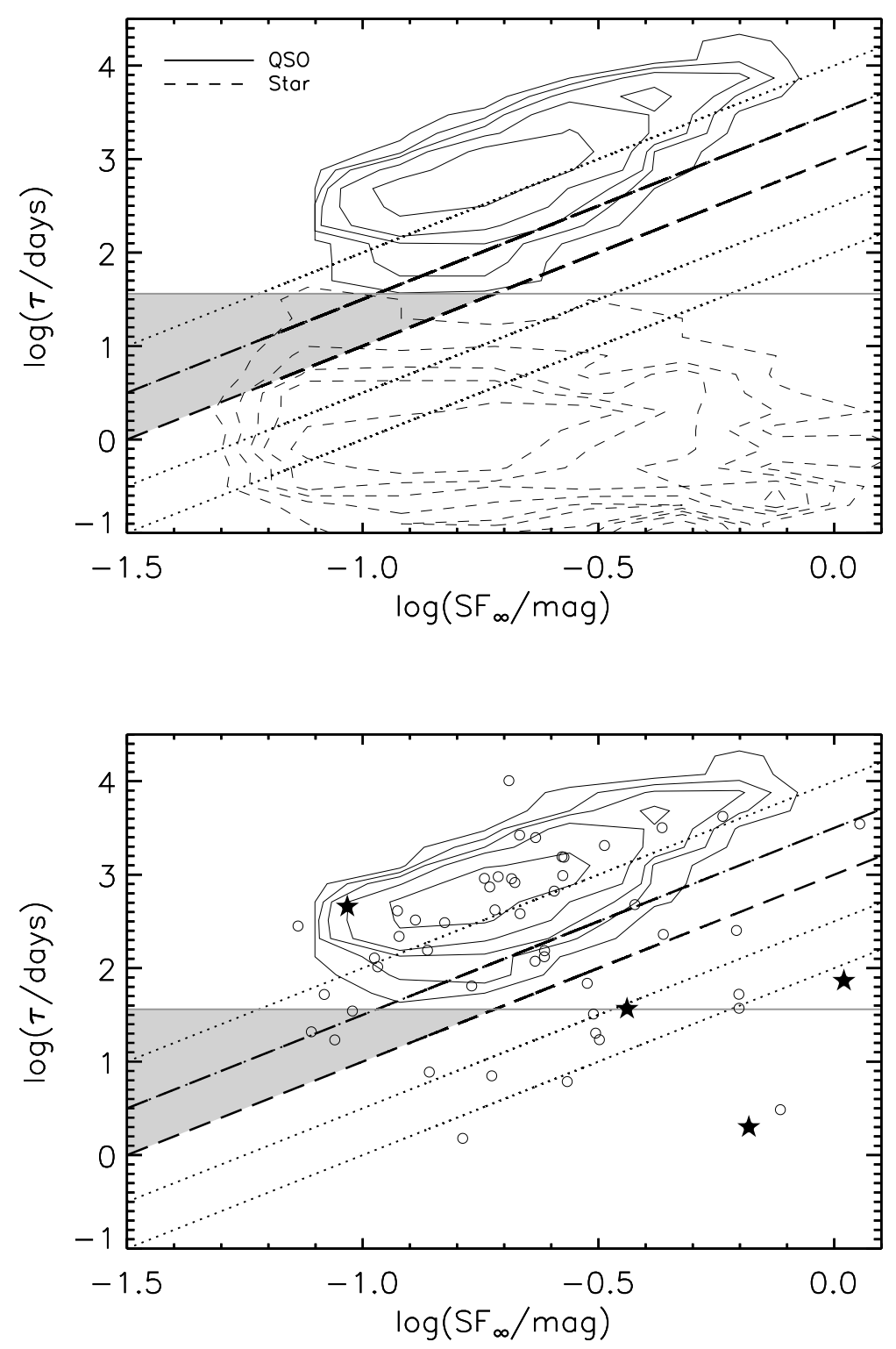

Fig. 13.- Top: Characteristic time scale $\tau$ versus asymptotic rms variability $\mathrm{SF}_{\infty}$ for confirmed quasars (solid) and stars (dashed) in our S82 data set restricted to $i<19$. The contours show regions containing $40,60,75,85$, and $90 \%$ of data points in each subsample. The dotted lines represent lines of constant $\hat{\sigma}=\mathrm{SF}_{\infty} / \sqrt{\tau}$. The two thick dashed lines correspond to $\hat{\sigma}=10^{-0.22}$ and $10^{-0.47} \mathrm{mag}^{-1 / 2}$. The gray region represents the contaminating (stellar) region when selecting sources with $\hat{\sigma}<10^{-0.22} \mathrm{mag}_{\mathrm{yr}}^{-1 / 2}$. When imposing a lower limit at $\tau=10^{1.56}$ days (gray horizontal line), these contaminants are excluded from the sample, leading to a higher efficiency of quasar selection. Bottom: As in top panel but for UVX objects only. Here, the confirmed stars are shown with filled stars, and UVX objects without SDSS spectra are shown with open circles. 


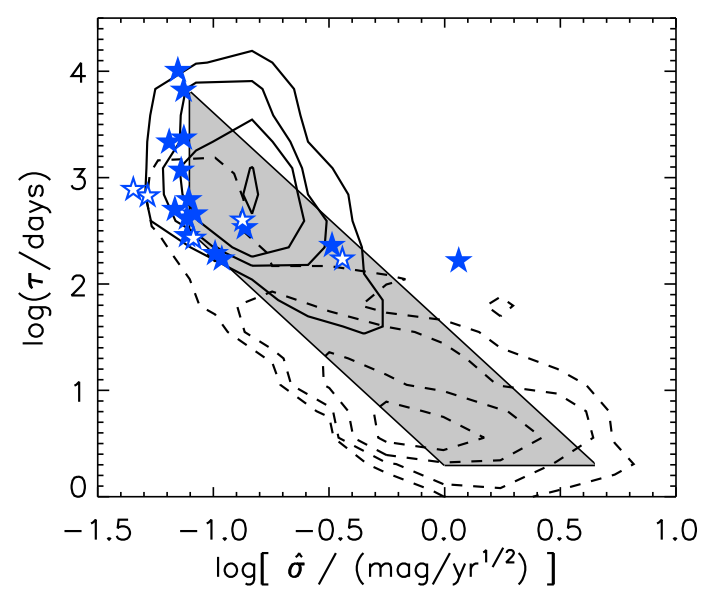

Fig. 14. - Variability time scale $\tau$ as a function of short-term driving amplitude $\hat{\sigma}$. The contours show the regions containing $25,50,75$, and $90 \%$ of data points for quasars (solid, $N=1170$ ) and all other objects (dashed, $N=1783$ ). The shaded region shows the quasar selection cut from Kozł10. The star symbols represent the confirmed stars passing our $\tau>100$ days and $\Delta L_{\text {noise }}>2$ selection criteria (except those flagged as having outliers in their light curves - see Table 1 caption). The star symbols filled with white indicate late-type stars (SDSS SpecClass=6).

\subsubsection{Comparison to Koztowski et al. (2010)}

Kozł10 proposed a quasar variability selection regions based on the light curves of OGLE-II and OGLE-III sources in the Magellanic Clouds with the mid-IR colors of AGN from Kozłowski \& Kochanek (2009) also with the requirement that $\Delta L_{\text {noise }}>2$. Figure 14 shows the distribution of spectroscopically identified quasars and other sources in this selection space as well as the confirmed stars passing the $\tau>100$ days and $\Delta L_{\text {noise }}>2$ selection cuts. The completeness of this selection region for the SDSS quasar sample is relatively low, $C=55 \%$, as is the efficiency, $E=46 \%$ (62\% when excluding outliers in light curves), which demands some explanation. The lower completeness is likely a consequence of the differences in the cadence and duration of the OGLE light curves as compared to the SDSS light curves. The OGLE light curves are densely sampled over the full durations 
of the surveys, and the typical OGLE-III light curve has 370 epochs over 7 years. As a result, fewer light curves have run-away estimates of $\tau$, making it relatively safe to impose an upper limit on $\tau$ in order to reduce contamination by long period variables (LPVs). In the very dense stellar environment of the Magellanic Clouds, the contamination by LPVs is significant. For the SDSS regions, if we simply eliminate the upper boundary of the Kozł10 selection region, the completeness rises to $C=91 \%$ with a corresponding rise in the efficiency to $E=52 \%$ (69\%, excluding outliers) because many more quasars are added than contaminating stars. Figure 14 does show significant contamination coming from sources with short time scales, and this was not observed in the Magellanic Cloud sample after removing the periodic variables (predominantly RR Lyrae and Cepheids). Part of the problem may again be the differences in the light curve cadences, where the better cadenced OGLE light curves can better distinguish $\tau \equiv 0$ from a small $\tau$, leading to less "leakage" of false sources past the $\Delta L_{\text {noise }}>2$ boundary. There are probably also some basic differences in the sources of contamination. Kozł10 were designing selection criterion for a small area $\left(1.3 \mathrm{deg}^{2}\right)$ containing a large number of luminous stars whose natural time scales are relatively long. Here, we are examining a much wider area $\left(213 \mathrm{deg}^{2}\right)$ where the variable stars are generally not as luminous and/or are white dwarfs, leading to relatively shorter natural time scales. Thus, the generous boundary for short $\tau$ in Kozł10 may not be appropriate to a wide area survey. If we raise the $\tau>2$ day lower limit in Kozł10 to $\tau>10^{1.5}$ days, then for the SDSS region the completeness changes little $(C=90 \%)$, but the efficiency rises dramatically to $E=78 \%$ (86\%, excluding outliers). Finally, if we consider the variability properties of the variable spectroscopically identified stars in this diagram, they tend to lie in the region of low amplitudes $\hat{\sigma}$ and long time scales. In the Magellanic Cloud regions, where Kozł10 could take advantage of color magnitude diagrams to better characterize the variable stars, these tend to be various classes of evolved, red stars - long period variables (LPV), long secondary period (LSP) variables and OGLE Small Amplitude 
Variable Red Giants (OSARGS).

\subsubsection{Comparison to Butler \& Bloom (2010)}

Butler \& Bloom (2010, hereafter B\&B) present a different approach to using the DRW model to select quasars based on variability. They model the ensemble SF for quasars (see, e.g., Vanden Berk et al. 2004) as a function of apparent magnitude with the DRW model, and use this to predict $\tau$ and $\hat{\sigma}$ for individual quasar candidates. The process model $\chi^{2}$ is then computed for each light curve using these fixed DRW parameters to obtain $\chi_{\mathrm{QSO}}^{2}$. Essentially, they fit the DRW model with a (infinitely) strong prior on the values of $\tau$ and $\hat{\sigma}$ derived from the apparent magnitude. They also compute $\chi_{\text {FALSE }}^{2}$, the goodness of fit expected if the same DRW model was fit to a light curve that was really white noise with the overall variance of the observed light curve. The main difference with respect to our method is that we obtain individual estimates of $\tau$ and $\hat{\sigma}$ for every light curve independent of the mean magnitude. This leads to a much wider distribution of $\tau$ and $\hat{\sigma}$ for our method with respect to $\mathrm{B} \& \mathrm{~B}$, allowing us to use $\tau$ as an efficient classifier. The selection technique used in B\&B is very efficient, however, because only a single model rather than a parameter fit is required.

Here we compare the resulting $C$ and $E$ from each method using identical samples of light curves. In Table 2 we compare the two methods for the light curve sample with $-35^{\circ}<\mathrm{RA}<50^{\circ}$ and $i<19$, where the $\mathrm{B} \& \mathrm{~B}$ method selects an object as a quasar candidate if

$$
\begin{aligned}
& \text { - } \chi_{\mathrm{FALSE}}^{2} / \nu>\chi_{\mathrm{QSO}}^{2} / \nu \\
& \text { - } \chi_{\mathrm{FALSE}}^{2} / \nu>3.1 \text { if } \mathrm{RA}>-20 \operatorname{deg}(5 \sigma \text { significance }) \\
& \text { - } \chi_{\mathrm{FALSE}}^{2} / \nu>4.4 \text { if } \mathrm{RA}<-20 \operatorname{deg}(7 \sigma \text { significance }) \text {, }
\end{aligned}
$$


where $\nu$ is the number of degrees of freedom. Comparing the fourth and eleventh rows, one can see that $C$ is higher by a few percent for the B\&B method whereas $E$ is slightly lower. When instead choosing $\tau>10^{1.5}$ days and $\Delta L_{\text {noise }}>10$, a similar completeness is achieved (96\%) with $E=87 \%$. Therefore, with some fine-tuning of parameters, the two methods can deliver samples with comparable $C$ and $E$, which is not too surprising since they rely on the same mathematical model to describe the light curve behavior. The B\&B method is faster if the only goal is to select quasars, but fitting for $\tau$ and $\hat{\sigma}$ then allows for further classification of the type of variability, as illustrated by the locations of different classes of variable stars in the parameter space (see Figure 9 in Kozł10).

In Table 4, we present the cross-correlation between our method and that in B\&B in terms of how many $i<19$ sources are selected using either technique. For this table, we define our selection criteria as $\tau \geq 10^{1.5}$ days and $\Delta L_{\text {noise }}>10$ (after omitting light curve outliers). We define the B\&B selection criteria as listed above. We then show the characteristics of the samples defined by passing both criteria, at least one of the criteria, failing both, and passing only one or the other. It is beneficial to use both methods to select quasars. One can achieve $E=90 \%$ in the absence of color selection when requiring both criteria, but $C=97 \%$ can be maintained when using either criteria for an $E$ of $82 \%$. In essence, some quasars have true $\tau$ and $\hat{\sigma}$ values too far from the prior defined by the apparent magnitude and so have reduced B\&B likelihoods, while for some other objects, the addition of the strong B\&B prior leads to better rejection of false positives and negatives.

\section{Effect of Cadence and Photometric Accuracy on Best-fit Variability Parameters: Prospects for LSST and Pan-STARRS}

One clear lesson from our comparison of our proposed SDSS selection criteria (applied to sparsely sampled, effectively shorter duration light curves in a wide area, extragalactic 
Table 4. Cross-Correlation with $\mathrm{B} \& \mathrm{~B}$ for $-35^{\circ}<\mathrm{RA}<50^{\circ}$ and $i<19$

\begin{tabular}{|c|c|c|c|c|c|c|c|c|c|c|c|}
\hline & Selected By & $N$ & $C$ & $\delta C$ & $E$ & $\delta E$ & Spec. & QSO & Star & Gal. & Unk. \\
\hline 1. & All Sources & 10024 & 100 & 4 & 15 & 0.4 & 33 & 45 & 53 & 2.5 & 0.15 \\
\hline 2. & Both Methods & 1564 & 95 & 3 & 90 & 3 & 92 & 98 & 1.3 & 0.35 & 0.14 \\
\hline 3. & Either Method & 1763 & 97 & 4 & 82 & 3 & 85 & 96 & 3.1 & 0.60 & 0.13 \\
\hline 4. & Neither Method & 8237 & 2.6 & 0.4 & 0.5 & 0.1 & 22 & 2.1 & 94 & 4.0 & 0.11 \\
\hline 5. & Only our Method & 83 & 0.9 & 0.3 & 17 & 5 & 37 & 45 & 39 & 13 & 3.2 \\
\hline 6. & Only B\&B & 138 & 1.5 & 0.3 & 16 & 4 & 33 & 49 & 49 & 2.2 & 2.2 \\
\hline
\end{tabular}

Note. - Column definitions follow those in Table 2, The second row lists results for a sample satisfying both the B\&B criteria as well as our criteria $\left(\tau \geq 10^{1.5}\right.$ days and $\Delta L_{\text {noise }}>10$, after omitting light curve outliers). The third row lists results for a sample satisfying either the $\mathrm{B} \& \mathrm{~B}$ criteria or ours. The selection in the fourth row is rejected by both methods, while the selections in the fifth and sixth rows only satisfy one of the methods. 
field) and the Kozł10 selection criteria (applied to densely sampled, longer duration light curves in a small area with high stellar densities) is that the appropriate selection criteria will depend on both the properties of the light curves (duration, cadencing, and uncertainties) and the nature of the contamination. Here we consider the effects that the survey length, time sampling, and photometric errors have on the best-fit DRW parameters. These are important issues to consider for upcoming surveys such as Pan-STARRS (Kaiser et al. 2002), which is already taking data, and LSST (Ivezić et al. 2008), which will obtain accurate, well-sampled light curves for millions of quasars. To probe the time scales as short as $0.1 \tau$, and assuming a rest-frame time scale of $\tau=10$ days and a characteristic redshift of 2 , the light curves should be sampled every 3 days in the observer's frame, which is in good agreement with the baseline cadence of LSST. The LSST photometric errors in the $r$ band will be $<0.02$ mag for $r<22$, and there are roughly 2-3 million AGN with $r<22$ in the 20,000 sq. deg. covered by the main LSST survey (see Table 10.2 in the LSST Science Book; LSST Science Collaborations and LSST Project 2009). Each of these objects will be observed about 1000 times (summed over all bands), yielding a database of over 2 billion photometric measurements. The characteristic time scale $\tau$ will be well-constrained if the survey length is sufficiently longer than $\tau$. It is therefore necessary to evaluate the accuracy of fitted variability parameters as a function of light curve length.

\subsection{The Impact of Light Curve Length}

As a first test, we generate 10,000 DRW light curves using an input of $\tau=575$ days and $\mathrm{SF}_{\infty}=0.2 \mathrm{mag}$, which are the median observed-frame values for the S82 quasar sample. The light curves are sampled once every 10 days $(\Delta t=10$ days $)$ and span a length of 40 years. Photometric errors are drawn from a Gaussian distribution with a standard deviation of $\sigma=0.01 \mathrm{mag}$. To test the impact of light curve length, we truncate the light curves to 
10 years, 3 years, and 1 year and compare the resulting variability parameters. For data lengths shorter than $\tau$, only the driving amplitude of short-term fluctuations, $\hat{\sigma}=\mathrm{SF}_{\infty} / \sqrt{\tau}$,

is well constrained [recall that $S F(\Delta t<<\tau)=\hat{\sigma} \sqrt{|\Delta t|}$. That is, on a grid of $\log (\tau)$ versus $\log \left(\mathrm{SF}_{\infty}\right)$, the best-fit values will be scattered due to fitting errors along lines of constant $\hat{\sigma}$, but much less perpendicular to it (i.e., along lines of constant $K=\tau \sqrt{\mathrm{SF}_{\infty}}$ ). Therefore, as the light curve length decreases, the mean best-fit $\hat{\sigma}$ will not vary significantly, while the best-fit parameters $\tau$ and $\mathrm{SF}_{\infty}$, and $K$ will become biased (see Kozł10; MacLeod et al. 2010). Note that only two of these four parameters are independent.

Figure 15 shows the distribution of best-fit variability parameters $\hat{\sigma}, K, \tau$, and $\mathrm{SF}_{\infty}$ normalized by the input values for simulated light curves with length 40 years, as well as when truncated to 10 years, 3 years, and 1 year. As the length increases, the widths of the output parameter distributions decrease, roughly as the square root of the light curve length. While the mean output value of $\hat{\sigma}$ is unaffected by the light curve length, the mean values of $K, \tau$, and $\mathrm{SF}_{\infty}$ decrease as the length shortens, and the number of "run-away" time scales $\left(\tau \geq 10^{5}\right.$ days) increases due to the fact that $\tau$ is now longer than the light curve length and cannot be constrained. We find that for typical variable quasars, the best-fit distributions of $\tau$ and $\mathrm{SF}_{\infty}$ are strongly biased for surveys significantly shorter than 10 years (by about $70 \%$ and $40 \%$, respectively, for a length of 3 years). Nevertheless, the $\hat{\sigma}$ parameter is unbiased and already well-measured ( $\mathrm{rms}=20 \%)$ after 1 year.

\subsection{The Impact of Realistic Cadence}

As a second test, we generate 10,000 light curves using a simulated $r$-band LSST cadence ( 200 observations spread over 10 years; Delgado et al. 2006). An example light curve with this cadence is shown in Figure 16. Figure 17 compares the resulting parameters for the LSST cadence and a cadence of $\Delta t=10$ days over 10 years as in the first test. 
The only significant change is an increased width for the best-fit $\hat{\sigma}$ distribution, where the accuracy of the best-fit $\hat{\sigma}$ drops from $5 \%$ to $13 \%$.

\subsection{The Impact of Photometric Accuracy}

As a third test, we evaluate the effect that the photometric accuracy has on the fitted parameters by generating 10,000 light curves with the $r$-band LSST cadence and photometric errors drawn from a Gaussian of width $\sigma=0.03$ mag. The same is done for $\sigma=0.1 \mathrm{mag}$. The output parameters for these two sets of light curves are compared to those from the second test, where $\sigma=0.01 \mathrm{mag}$, in Figure 18. As expected, the distributions become broader as the photometric error bars increase and the fits become more uncertain. While a photometric accuracy of $\sigma=0.1$ mag leads to a significant increase in the $\hat{\sigma}$ errors compared to the $\sigma=0.01 \mathrm{mag}$ case (a factor of $4 \mathrm{dex}$ ), an accuracy of $\sigma=0.03 \mathrm{mag}$ leads

to only a marginally wider distribution (40\%). We conclude that a photometric accuracy of $\sigma=0.03 \mathrm{mag}$ is sufficient to obtain well-constrained variability parameters for typical quasars.

\subsection{Selection Completeness for a Realistic LSST Simulation}

As our final test, we estimate the completeness of quasar selection based on the $\tau>100$ days criterion alone for three different light curve lengths (1, 3, and 10 years), the $r$-band LSST cadence, and a photometric accuracy of $\sigma=0.03$ mag (achieved at $i \approx 22$ ). Now, instead of using fixed input $\tau$ and $\mathrm{SF}_{\infty}$ values, we use the full observed $r$-band distributions for the S82 quasar sample as presented in MacLeod et al. (2010). The distribution of input $\tau$ is shown as the gray histogram in the top panel of Figure 19. The output distributions for each light curve length are also shown. We compute the 
completeness as the fraction of the output distribution with a best-fit $\tau$ exceeding 100 days. The completeness for the input distribution is $91 \%$ (different than the $94 \%$ reported in Table 2 because all S82 quasars are considered, without rms $>0.05$ or $i<19$ mag limits). For light curve lengths of 10,3 , and 1 year(s), $C$ is estimated to be $88 \%, 75 \%$, and $51 \%$, respectively. Notably, with 10 years of observations, imperfectly measured $\tau$ causes only $\sim 3 \%$ of the sample to "leak" below the $\tau=100$ day boundary. Because it is not known what the contamination will be like for a typical LSST field, we cannot estimate the efficiency for the $\tau>100$ days selection. With the aid of multi-band imaging, colors can be used along with a relaxed $\tau$ cut to obtain highly complete samples early in the survey $(\sim 1$ year).

While the light curve length clearly has an impact on the best-fit time scale distribution, we also wish to estimate the effect of different cadences in general. This is motivated by the fact that Kozł10 found a large fraction of quasars concentrated at smaller $\tau$ when applying the DRW model to OGLE-II and OGLE-III light curves, which are more densely-sampled than the SDSS S82 data. Also, the Pan-STARRS1 $3 \pi$ survey will obtain 6 epochs in a given band over 3 years, as opposed to 60 epochs for LSST. Therefore, we wish to determine whether a $\tau$ selection can still deliver decently complete samples of quasars in a typical 3 year-long Pan-STARRS survey. We use Table 4 in Schm10 to simulate a typical Pan-STARRS cadence and assume that the griz bands can be combined to give 24 total epochs over 3 years (we omit the $Y$ band because of its lower sensitivity).

To make the comparison, we use a uniform input distribution of $\log (\tau)$ and $\log \left(\mathrm{SF}_{\infty}\right)$ within the region defined by $2<\log (\tau /$ days $)<3.5$ and $-1.4<\log \left(\mathrm{SF}_{\infty} / \mathrm{mag}\right)<-0.2$. We do not use the observed $\tau$ distribution for SDSS quasars for the input, as in the previous test, in order to ignore any potential effects of a biased $\tau$ distribution caused by the SDSS sampling. We compare the fits at a fixed photometric uncertainty (0.03 mag), therefore 
assuming that the absolute depth of each survey does not play a role in these comparisons 4 . In the middle and bottom panels of Figure 19, we compare the completeness results for the proposed LSST samplings to the typical 10 year-long S82, 7 year-long OGLE-III, and 3 year-long (24-epoch) Pan-STARRS samplings. For each cadence, we compute the completeness as the fraction of best-fit $\tau$ that does not "leak" below the $\tau=100$ day boundary. The results show that the denser time sampling of the OGLE-III survey leads to a higher completeness and a more compact spread in output $\tau$ when compared to S82, and a similar completeness when compared to the 10-year $r$-band LSST cadence ( 93\%). We note that the time scales for the minority of OGLE-III objects that also have OGLE-II light curves will be even better constrained, since for these the total duration is $\sim 10$ years. For Pan-STARRS1 3 $\pi, C$ is estimated to be $70 \%$ for $\tau>100$ days after combining the four griz bands. This is comparable to the result for a 3-year $r$-band LSST cadence. For a single Pan-STARRS band (6 epochs over 3 years), $C$ will likely be significantly worse, so in this case an alternate selection method (such as one based on $\hat{\sigma}$ ) may be optimal in the absence of color selection.

\subsubsection{Comparison to Butler \& Bloom (2010)}

We carried out similar simulations for the B\&B method, using the same uniform input distribution of $\log (\tau)$ and $\log \left(\mathrm{SF}_{\infty}\right)$. Based on the mean trend of $\hat{\sigma}^{2}$ with g magnitude found for the S82 quasar sample in MacLeod et al. (2010), we assigned each light curve an

\footnotetext{
${ }^{4}$ Because the LSST survey has a fainter magnitude limit than OGLE, SDSS, or Pan-STARRS, it will have a higher completeness for quasar selection. Since we wish to compare differences due to cadencing only, we compare at a fixed photometric uncertainty. We note that a fixed uncertainty will induce some differences in the $\tau$ distribution between surveys from what would realistically be observed. However, that is a higher order question than what is examined here.
} 
apparent magnitude of

$$
g=0.334 \log \hat{\sigma}^{2}+21.38+G(0.8)
$$

where $G(0.8)$ is a Gaussian random deviate of width 0.8 mag, matching the observed scatter about the mean trend.

Figure 20 shows the output distributions of $\chi_{\mathrm{FALSE}}^{2} / \nu$ and $\chi_{\mathrm{QSO}}^{2} / \nu$, as well as the B\&B selection limits for a $5 \sigma$ significance cut against false positives. The figure legends list the cadences and the resulting completeness $C$. As survey length increases, $C$ increases because of an overall increase in $\chi_{\text {FALSE }}^{2} / \nu$. For the single-year LSST cadence, $C$ is only $20 \%$, and we find a similar result for the 3-year single-band Pan-STARRS $3 \pi$ cadence. When combining the Pan-STARRS griz bands, $C$ increases to $52 \%$, slightly lower than the 3-year LSST cadence. The LSST 10-year completeness is lower than what was found when choosing $\tau \geq 100$ days in the previous section (86\% vs. $92 \%$ ), but it could be increased by weakening the cut on $\chi_{\mathrm{FALSE}}^{2} / \nu$. However, because the contamination rate is not known for LSST, it is unclear as to how much this cut could be varied without a major decrease in $E$. Again, the OGLE cadence leads to the highest $C$ due to the large number of epochs. We also find a significant decrease in $C$ for the $\mathrm{S} 82$ cadence from what is observed in the eleventh row of Table 2 ( $81 \%$ vs. $96 \%$ ). This may be due to the prescription for generating mean magnitudes. A larger $C$ is expected if we were to use the scaling in Table 1 in B\&B, because the $\chi_{\text {FALSE }}^{2} / \nu$ would likely be larger overall (i.e., white noise would be less likely). 

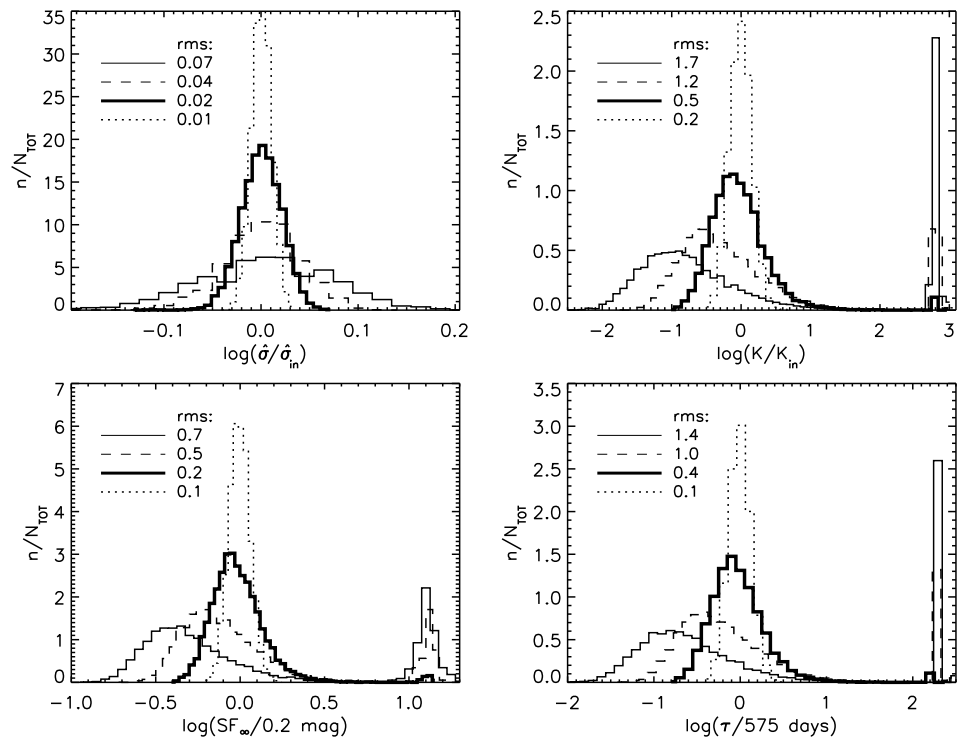

Fig. 15. - Distributions of $\hat{\sigma}=\mathrm{SF}_{\infty} / \sqrt{\tau}, K=\tau \sqrt{\mathrm{SF}_{\infty}}, \tau$, and $\mathrm{SF}_{\infty}$ normalized by the input values for 10,000 simulated light curves with length 40 years (dotted), 10 years (thick), 3 years (dashed), and 1 year (thin solid), all using $\Delta t=10$ days and photometric accuracy of 0.01 mag. $n$ indicates the number of points in a bin divided by the bin width, and $N_{T O T}$ is the total number of points used for each histogram. The rms of each distribution is listed in the legend.

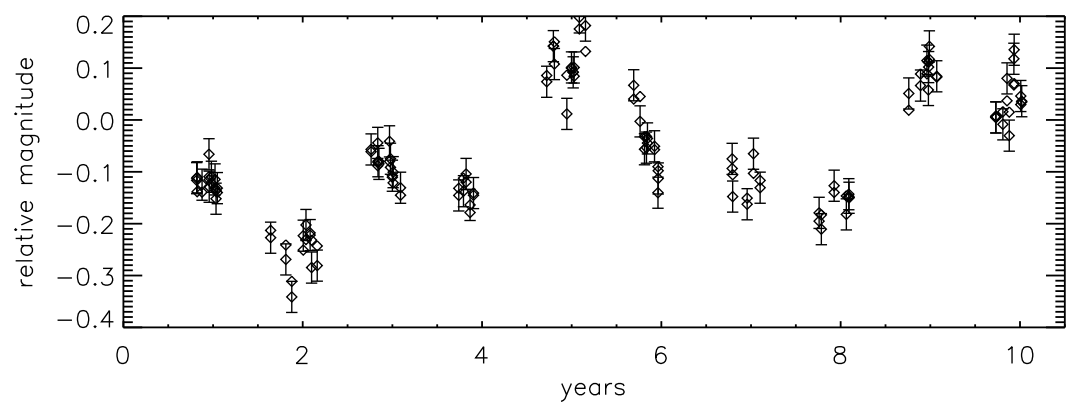

Fig. 16. - A model light curve generated for $\tau=575$ days, $\mathrm{SF}_{\infty}=0.2 \mathrm{mag}$, the $r$-band LSST cadence, and a photometric accuracy of 0.03 mag. 

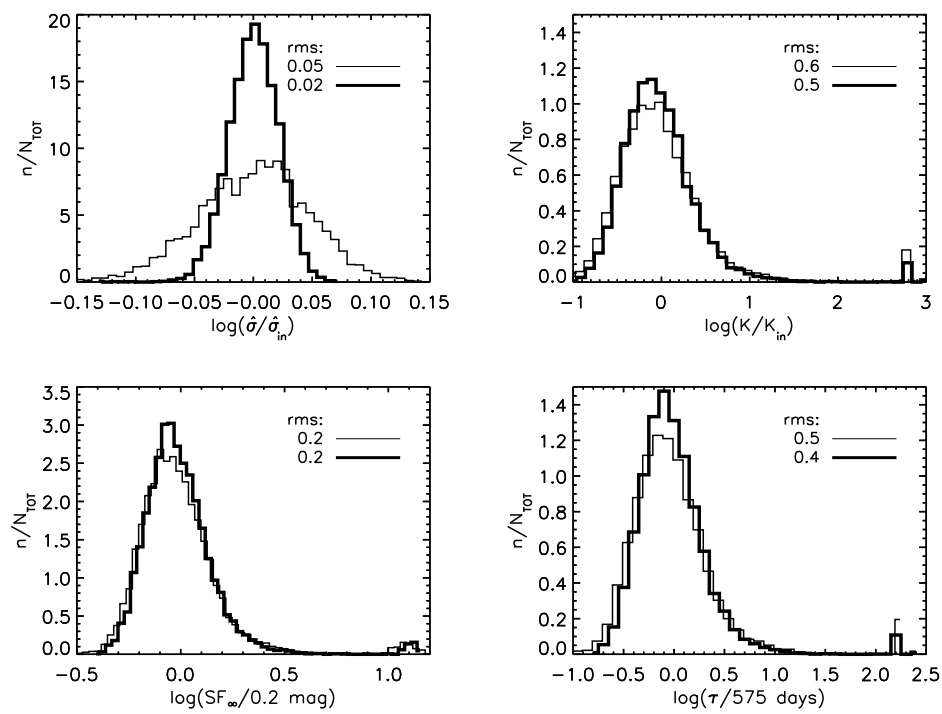

Fig. 17.- Distributions of $\hat{\sigma}=\mathrm{SF}_{\infty} / \sqrt{\tau}, K=\tau \sqrt{\mathrm{SF}_{\infty}}, \tau$, and $\mathrm{SF}_{\infty}$ normalized by the input values for 10,000 simulated light curves with a photometric accuracy of $0.01 \mathrm{mag}$ and the $r$-band LSST cadence with $\sim 200$ data points (thin solid). The thick line shows the distribution from Figure 15 with a cadence of $\Delta t=10$ days over 10 years (365 data points). The rms of each distribution is listed in the legend.
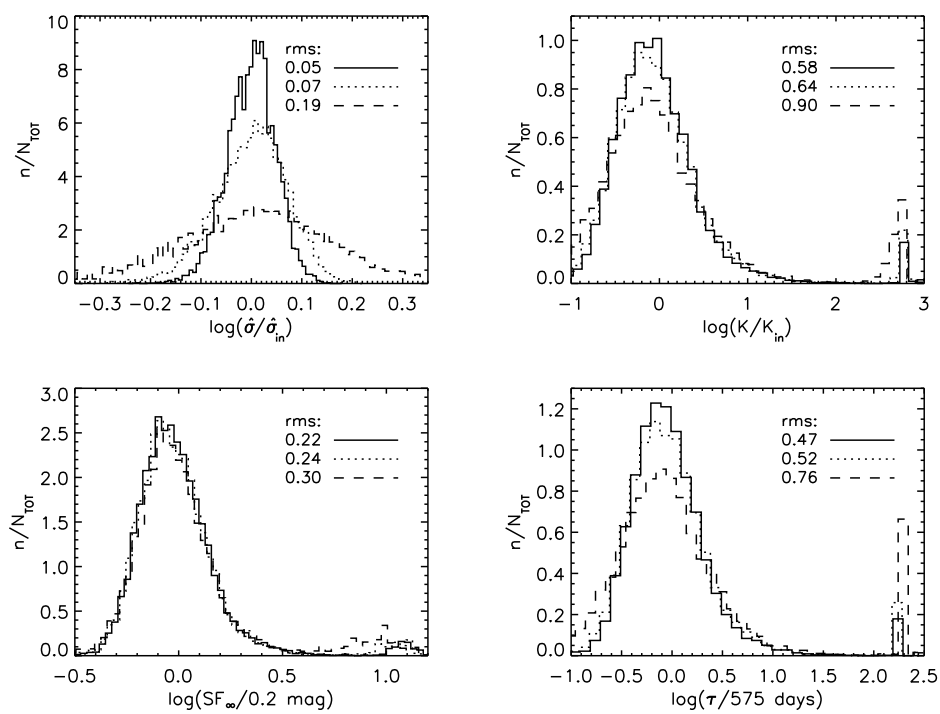

Fig. 18.- Distributions of $\hat{\sigma}=\mathrm{SF}_{\infty} / \sqrt{\tau}, K=\tau \sqrt{\mathrm{SF}_{\infty}}, \tau$, and $\mathrm{SF}_{\infty}$ normalized by the input values for 10,000 simulated light curves with a 10-year $r$-band LSST cadence and a photometric accuracy of 0.01 mag (solid), $0.03 \mathrm{mag}$ (dotted), and $0.1 \mathrm{mag}$ (dashed). The rms of each distribution is listed in the legend. 


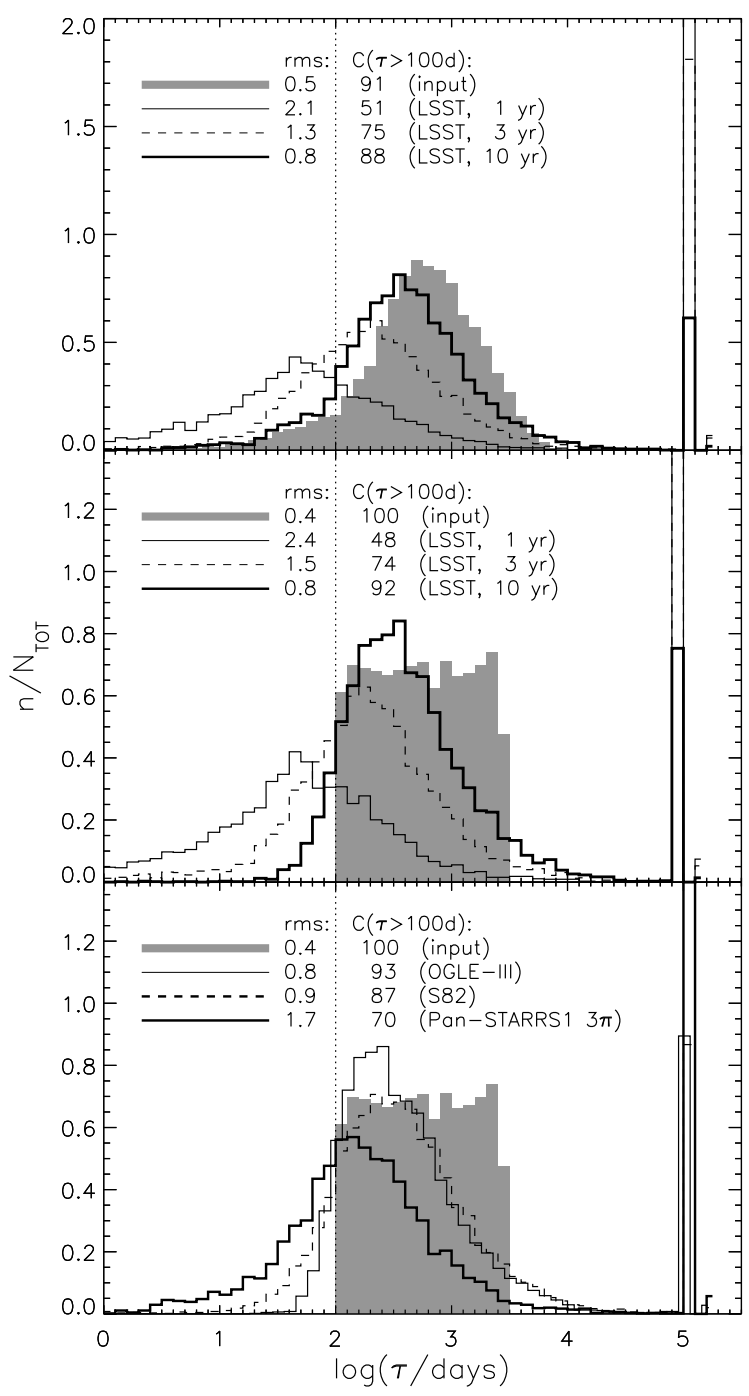

Fig. 19.- Top: Distributions of $\tau$ as compared to the input distribution (shaded histogram) for $\sim 7,000$ simulated light curves with the $r$-band LSST cadence, a photometric accuracy of 0.03 mag, and lengths of 1 year (thin solid), 3 years (dashed), and 10 years (thick solid). The rms of each distribution is listed in the legend, as well as the completeness of the quasar selection criteria $\tau \geq 100$ days (dotted line). Note that light curves significantly longer than 1 year are required to achieve $>50 \%$ completeness. Middle: As in top panel but for a uniform input distribution of $\tau$ within the range $100<\tau<10^{3.5}$ days. Bottom: As

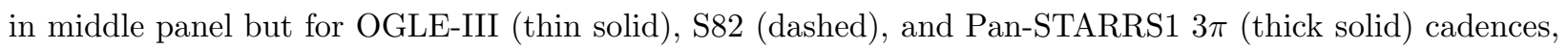
which span 7, 10, and 3 years, respectively. The denser time sampling of the OGLE-III survey leads to a higher completeness than S82. 


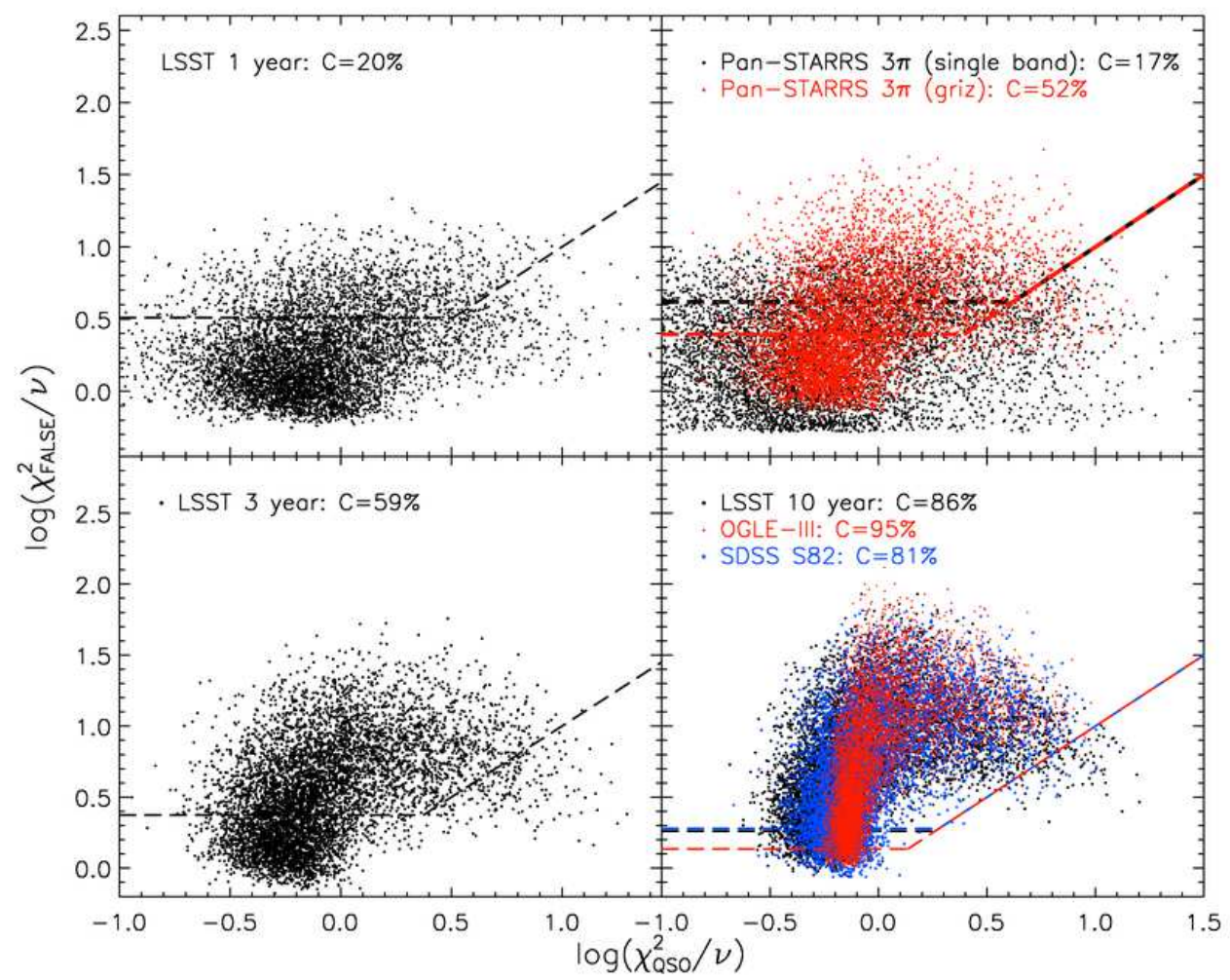

Fig. 20.- Distributions of $\chi_{\mathrm{FALSE}}^{2} / \nu$ and $\chi_{\mathrm{QSO}}^{2} / \nu$ resulting from fitting $\sim 7,000$ simulated DRW light curves with various cadences (listed in the legends) using the $\mathrm{B} \& \mathrm{~B}$ technique. The completeness of the quasar selection criteria (the fraction above the dashed lines) for each cadence is listed.

\section{Discussion and Summary}

We used the light curves for variable point sources from SDSS Stripe 82 to determine how well quasars can be separated from stars with the aid of photometric variability. The Stripe 82 data set, designed to search for supernovae, is excellent for quasar variability studies since it provides light curves for a large statistical sample of objects to a faint limiting magnitude (see Boutsia et al. 2010 for another example of using supernova surveys to search for quasars). The data set used here contains precise photometry (using recalibrated light curves by Ivezić et al. 2007 and Sesar et al. 2007), as well as extensive spectroscopy, which has enabled us to develop an efficient method for selecting quasars 
while maintaining high completeness. The variability model used here (a damped random walk, see KBS09; Kozł10) is robust enough to handle sparse or irregularly-sampled data. By modeling the variability as a stochastic process described by the exponential covariance matrix $S_{i j}=\sigma^{2} \exp \left(-\left|t_{i}-t_{j}\right| / \tau\right)$, only $\mathrm{O}(N)$ operations are required to determine the model parameters for a light curve with $N$ data points. This is an important algorithmic feature in the context of upcoming massive synoptic surveys, such as LSST.

The DRW model provides unique information on the characteristic time scale, $\tau$, for which a range has been observed for quasars (Sesar et al. 2006; KBS09; Kozł10; MacLeod et al. 2010). Compared to selecting solely on the short-term behavior of the SF (the parameter $\hat{\sigma}$ ), the inclusion of $\tau$ information boosts $C$ from $90 \%$ to $97 \%$ while maintaining an efficiency (purity, $E$ ) at $80 \%$, based on variability alone. Conversely, for $C=98 \%$, the $\tau$ information boosts $E$ from about $60 \%$ to $75 \%$, while at $C=90 \%, E$ rises from about $80 \%$ to $85 \%$. This method enables the selection of $z \sim 3$ quasars, where color selection largely fails. With the aid of $u-g$ and $g-r$ color selection, the efficiency can be further boosted to $96 \%$ with a reasonably high completeness of $93 \%$. It is worth reiterating that the $E$ determined here is only a lower limit because the spectroscopically confirmed quasar sample is not $100 \%$ complete. This performance is accomplished simply by imposing a lower limit on $\tau$ and an upper limit on $\hat{\sigma}$. The separation might improve when using more advanced techniques such as kernel density estimation (e.g., Richards 2008) rather than imposing simple limits.

By using the parametrization $S F(\Delta t)=A(\Delta t / 1 \mathrm{yr})^{\gamma}$, Schm10 was able to select quasars from UVX objects with $E \simeq 95 \%$ at $90 \%$ completeness when selecting on $A$ and $\gamma$. This selection is similar to one using $\hat{\sigma}$ alone, and provides a reasonable method for selecting quasars from short $(\lesssim 1$ year) light curves. With longer light curves, the inclusion of $\tau$ information significantly improves the performance. The differences in $C$ and $E$ are 
summarized in the bottom panels of Figure 12 and illustrated in Figure 13. If we restrict our initial sample to UVX objects and simply use $\tau \geq 100$ days to select quasars, we find that $C$ improves from the $90 \%$ found by Schm10 to $95 \%$, while $E$ improves from about $95 \%$

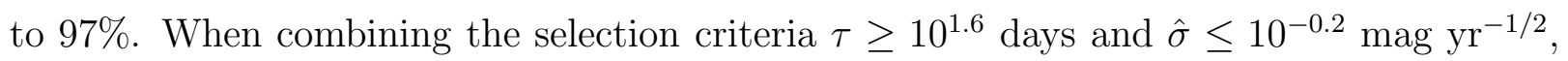
we find that $C$ improves further to $98 \%$ with $E=97 \%$. We note that the DRW model has benefits beyond the improved selection efficiency compared to Schm10. In addition to being a faster algorithm $\left[\mathrm{O}(N)\right.$ rather than $\left.\mathrm{O}\left(N^{2}\right)\right]$, the DRW model also produces information on the physics of the accretion disk since $\tau$ and $\mathrm{SF}_{\infty}$ are physical parameters that show correlations with luminosity, black hole mass, and wavelength (see MacLeod et al. 2010) and provides an approach for classifying other aperiodic sources as well (see Kozł10). The latter two points are also advantages of our approach over that in B\&B. For quasar selection, both methods yield similar results for completeness and efficiency.

Our selection criteria identify 255 objects with $i<19$ and $-35^{\circ}<\mathrm{RA}<50^{\circ}$ that vary on long time scales $\left(\tau>100\right.$ days with $\left.\Delta L_{\text {noise }}>2\right)$ and do not presently have SDSS spectra; the majority of them are likely quasars. Based on their light curves, several are expected to be interesting long-time scale variable objects, such as AGB stars. We plan to obtain follow-up spectroscopy for these objects (Table 1) with the goals of either confirming their quasar nature or identifying the major contaminants in quasar selection based on variability.

The DRW provides a complete statistical model for quasar variability. This is especially useful for evaluating the success of quasar selection in upcoming surveys, and enables us to estimate the completeness for different survey lengths, cadences, and photometric accuracy. We cannot estimate $E$, however, since it is dependent on an unknown makeup of the faint variable optical sky. For a typical (simulated) LSST cadence over 10 years and a photometric accuracy of $0.03 \mathrm{mag}$ (achieved at $i \approx 22$ ), $C$ is expected to be $88 \%$ 
for a sample selection criterion of $\tau>100$ days. For typical variable quasars, the best-fit distributions of $\tau$ and $\mathrm{SF}_{\infty}$ are biased for surveys shorter than 10 years (by about $70 \%$ and $40 \%$, respectively, for a length of 3 years). However, $\hat{\sigma}$ is well-constrained, and with the aid of multi-band imaging, colors can be used along with a relaxed $\tau$ cut to obtain highly complete samples early in the LSST survey ( $\sim$ year). For the Pan-STARRS1 $3 \pi$ survey, $C$ is estimated to be $70 \%$ for a $\tau>100$ day selection after combining the griz bands, which is comparable to the result for a 3-year $r$-band LSST cadence. The dense cadence of the LSST will provide tighter constraints on $\tau$ for a survey length of 10 years. This improvement is also important for relating observations to the physics of accretion disks (see MacLeod et al. 2010, and references therein). The most important conclusion of this work is that, given an adequate survey cadence, photometric variability provides an even better method than color selection for separating quasars from stars.

We acknowledge support by NSF grant AST-0807500 to the University of Washington, and NSF grant AST-0551161 to LSST for design and development activity. CSK and SK acknowledge support by NSF grants AST-0708082 and AST-1009756. RRG gratefully acknowledges support from NASA Chandra grants AR9-0015X and AR0-11014X.

Funding for the SDSS and SDSS-II has been provided by the Alfred P. Sloan Foundation, the Participating Institutions, the National Science Foundation, the U.S. Department of Energy, the National Aeronautics and Space Administration, the Japanese Monbukagakusho, the Max Planck Society, and the Higher Education Funding Council for England. The SDSS Web Site is http://www.sdss.org/.

The SDSS is managed by the Astrophysical Research Consortium for the Participating Institutions. The Participating Institutions are the American Museum of Natural History, Astrophysical Institute Potsdam, University of Basel, University of Cambridge, Case Western Reserve University, University of Chicago, Drexel University, Fermilab, the 
Institute for Advanced Study, the Japan Participation Group, Johns Hopkins University, the Joint Institute for Nuclear Astrophysics, the Kavli Institute for Particle Astrophysics and Cosmology, the Korean Scientist Group, the Chinese Academy of Sciences (LAMOST), Los Alamos National Laboratory, the Max-Planck-Institute for Astronomy (MPIA), the Max-Planck-Institute for Astrophysics (MPA), New Mexico State University, Ohio State University, University of Pittsburgh, University of Portsmouth, Princeton University, the United States Naval Observatory, and the University of Washington. 


\section{REFERENCES}

Abazajian, K. N., et al. 2009, ApJS, 182, 543

Agüeros, M. A. et al. 2005, AJ, 130, 1022

Boutsia, K., Leibundgut, B., Trevese, D., \& Vagnetti, F. 2009, A\&A, 497, 81

Butler, N. R., \& Bloom, J. S. 2010, arXiv:1008.3143 [B\&B]

de Vries, W. H., Becker, R. H., White, R. L., \& Loomis, C. 2005, AJ, 129, 615

Delgado, F., Cook, K., Miller, M., Allsman, R., \& Pierfederici, F. 2006, Proc. SPIE, 6270, $62701 \mathrm{D}$

Fukugita, M., Ichikawa, T., Gunn, J.E., Doi, M., Shimasaku, K., \& Schneider, D.P. 1996, AJ, 111, 1748

Gunn, J.E., et al. 1998, AJ, 116, 3040

Hennawi, J. F., \& Prochaska, J. X. 2007, ApJ, 655, 735

Ivezić, Ž., et al. 2007, AJ, 134, 973

Ivezić, Ž., Tyson, J. A., Allsman, R., Andrew, J., Angel, R., \& for the LSST Collaboration 2008, arXiv:0805.2366

Jiang, L., et al. 2006, AJ, 131, 2788

Kaiser, N., et al. 2002, Proc. SPIE, 4836, 154

Kelly, B. C., Bechtold, J., \& Siemiginowska, A. 2009, ApJ, 698, 895 [KBS09]

Kimball, A. E., \& Ivezić, Ž. 2008, AJ, 136, 684

Kollmeier, J. A., et al. 2006, ApJ, 648, 128 
Kozłowski, S., et al. 2010a, ApJ, 708, 927 [Kozł10]

LSST Science Collaborations and LSST Project 2009, LSST Science Book, Version 2.0, arXiv:0912.0201, http://www.lsst.org/lsst/scibook

MacLeod, C. L., et al. 2010, ApJ, 721, 1014

McDonald, P., \& Eisenstein, D. J. 2007, Phys. Rev. D, 76, 063009

McHardy, I. 2010, Lecture Notes in Physics, Berlin Springer Verlag, 794, 203

Press, W. H., Rybicki, G. B., \& Hewitt, J. N. 1992, ApJ, 385, 404

Richards, G. T., et al. 2002, AJ, 123, 2945

Richards, G. T. 2008, American Institute of Physics Conference Series, 1082, 22

Richards, G. T., et al. 2009, ApJS, 180, 67

Reimann, J. D. 1994, Ph.D. Thesis

Ross, N. P., et al. 2009, ApJ, 697, 1634

Rybicki, G. B., \& Press, W. H. 1992, ApJ, 398, 169

Rybicki, G. B., \& Press, W. H. 1994, Computer, 5004

Schlegel, D. J., Finkbeiner, D. P., \& Davis, M. 1998, ApJ, 500, 525

Schmidt, K. B., Marshall, P. J., Rix, H.-W., Jester, S., Hennawi, J. F., \& Dobler, G. 2010, ApJ, 714, 1194 [Schm10]

Schneider, D. P., et al. 2007, AJ, 134, 102

Schneider, D. P., et al. 2010, AJ, 139, 2360 
Sesar, B., et al. 2006, AJ, 131, 2801

Sesar, B., et al. 2007, AJ, 134, 2236

Vanden Berk, D. E., et al. 2004, ApJ, 601, 692

Voges, W., et al. 1999, A\&A, 349, 389

York, D.G. et al. 2000, AJ, 120, 1579 